\title{
A taxonomic monograph of the liphistiid spider genus Heptathela, endemic to Japanese islands
}

\author{
Xin Xu ${ }^{1,2}$, Hirotsugu Ono ${ }^{3}$, Matjaž Kuntnerre, ${ }^{2,45,6}$, Fengxiang $\mathrm{Liu}^{2}$, Daiqin $\mathrm{Li}^{7}$ \\ I College of Life Sciences, Hunan Normal University, 36 Lushan Road, Changsha 410081, Hunan Province, \\ China 2 State Key Laboratory of Biocatalysis and Enzyme Engineering, Centre for Behavioural Ecology and \\ Evolution, School of Life Sciences, Hubei University, 368 Youyi Road, Wuhan 430062, Hubei Province, China \\ 3 Department of Zoology, National Museum of Nature and Science, 4-1-1 Amakubo, Tsukuba-shi, Ibaraki- \\ ken, 305-0005, Japan 4 Evolutionary Zoology Laboratory, Department of Organisms and Ecosystems Research, \\ National Institute of Biology, Ljubljana, Slovenia 5 Evolutionary Zoology Laboratory, Biological Institute ZRC \\ SAZU, Ljubljana, Slovenia 6 Department of Entomology, National Museum of Natural History, Smithsonian \\ Institution, Washington, DC, USA 7 Department of Biological Sciences, National University of Singapore, 14 \\ Science Drive 4, 117543, Singapore \\ Corresponding authors: Xin Xu (xuxin_09@163.com); Daiqin Li (dbslidq@nus.edu.sg)
}

Academic editor:Jeremy Miller | Received 13 March 2019 | Accepted 5 September 2019 | Published 11 November 2019

http://zoobank.org/B995C056-97EC-41A4-9012-B58F9D3AFDC1

Citation: Xu X, Ono H, Kuntner M, Liu F, Li D (2019) A taxonomic monograph of the liphistiid spider genus Heptathela, endemic to Japanese islands. ZooKeys 888: 1-50. https://doi.org/10.3897/zookeys.888.34494

\begin{abstract}
Among the eight extant genera of primitively segmented spiders, family Liphistiidae, two are confined to East Asian islands, Heptathela Kishida, 1923 and Ryuthela Haupt, 1983. In this paper, a taxonomic revision of the genus Heptathela (Heptathelinae) from Kyushu and Ryukyu archipelago, Japan is provided. This study follows a multi-tier species delimitation strategy within an integrative taxonomic framework that is presented in a parallel paper, in which diagnosable lineages are considered as valid species. There, the initial hypothesis of species diversity (19) based on classical morphological diagnoses is tested with multiple species delimitation methods aimed at resolving conflict in data. This revision follows those analyses that converge on the species diversity of 20 , which includes a pair of cryptic species that would have been undetected with morphology alone. After this revision, eight previously described species remain valid, two junior synonyms are proposed, and 12 new Heptathela species are described based on diagnostic evidence. To ease identification and to hint at putative evolutionary units, Heptathela is divided into three groups. The Kyushu group contains H. higoensis Haupt, 1983, H. kikuyai Ono, 1998, H. kimurai (Kishida, 1920), and H. yakushimaensis Ono, 1998; the Amami group contains H. amamiensis Haupt, 1983, H. kanenoi Ono, 1996, H. kojima sp. nov., H. sumiyo sp. nov., and H. uken sp. nov.; and the Ok-
\end{abstract}

Copyright $\mathrm{Xin} X u$ et al. This is an open access article distributed under the terms of the Creative Commons Attribution License (CC BY 4.0), which permits unrestricted use, distribution, and reproduction in any medium, provided the original author and source are credited. 
inawa group contains $H$. yanbaruensis Haupt, 1983, H. aha sp. nov., H. gayozan sp. nov., H. kubayama sp. nov., $H$. mae sp. nov., $H$. otoha sp. nov., $H$. shuri sp. nov., $H$. tokashiki sp. nov., H. unten sp. nov., and H. crypta sp. nov. Heptathela helios Tanikawa \& Miyashita, 2014 is not assigned to a species group. A combination of diagnostic tools augments the morphological diagnoses that, in isolation, would be prone to error in morphologically challenging groups of organisms.

\section{Keywords}

Heptathelinae, island endemism, Kyushu, Ryukyu archipelago, species delimitation, trapdoor spiders

\section{Introduction}

The spider family Liphistiidae is the sole extant lineage within the ancient suborder Mesothelae known to possess a combination of plesiomorphic traits, for example, abdominal tergites (Fig. 1A), as well as spinnerets that have a mid-venter position (Fig. 1B). Compared to its sister clade that comprises all other spider families, liphistiids are genus- and species-poor, containing eight genera restricted to Southeast and East Asia (Xu et al. 2015a, b; World Spider Catalog 2019). Only two of these genera are purely insular, both being restricted to Japanese islands, and we focus here on the taxonomy of the nominal genus Heptathela Kishida, 1923 (Heptathelinae) (Fig. 1A, B).

Taxonomic limits of Heptathela were recently redefined (Xu et al. 2015b) as its taxonomic history that we briefly summarise here had been inconclusive. Kishida (1923) named the genus Heptathela for Liphistius kimurai Kishida, 1920 because its female had seven spinnerets, unlike the expected eight as in other species of Liphistius Schiödte, 1849. Although originally placed in Liphistiidae, Heptathela was once considered a family rank taxon, Heptathelidae (Petrunkevitch, 1939), again stressing the spinneret number difference with Liphistiidae. Only later, it was clarified that the spinneret number is variable among Heptathela species and not suitable for high level taxonomic diagnosis, even if it is stable in Liphistius (Yoshikura 1955; Haupt 1983, 2003; Xu et al. 2015a, b). Nonetheless, the genus name remained to be used for many "non-Liphistius" liphistiid lineages until 1983 when the second genus, Ryuthela Haupt, 1983, was added to the family Heptathelidae (Haupt 1983). This major clade was subsequently stabilised as the liphistiid subfamily Heptathelinae (Ono 2000; Xu et al. 2015b), but with several additional genera. Both Ono (2000) and Haupt (2003) relimited Heptathela to apply only to the species inhabiting the Japanese island Kyushu as well as the northern Ryukyu archipelago. These authors agreed that the species from China and Vietnam belonged to distinct genera. They disagreed, however, in the details. While Ono (2000) placed the mainland heptathelines into three genera (Abcathela, Vinathela, and Songthela), Haupt (2003) rejected Abcathela and Vinathela, erected instead Nanthela and Sinothela, and proposed Songthela as a synonym of the latter. To complete the full circle of this complex taxonomic history, Schwendinger and Ono (2011) reverted to only two heptatheline genera. According to these authors, all 


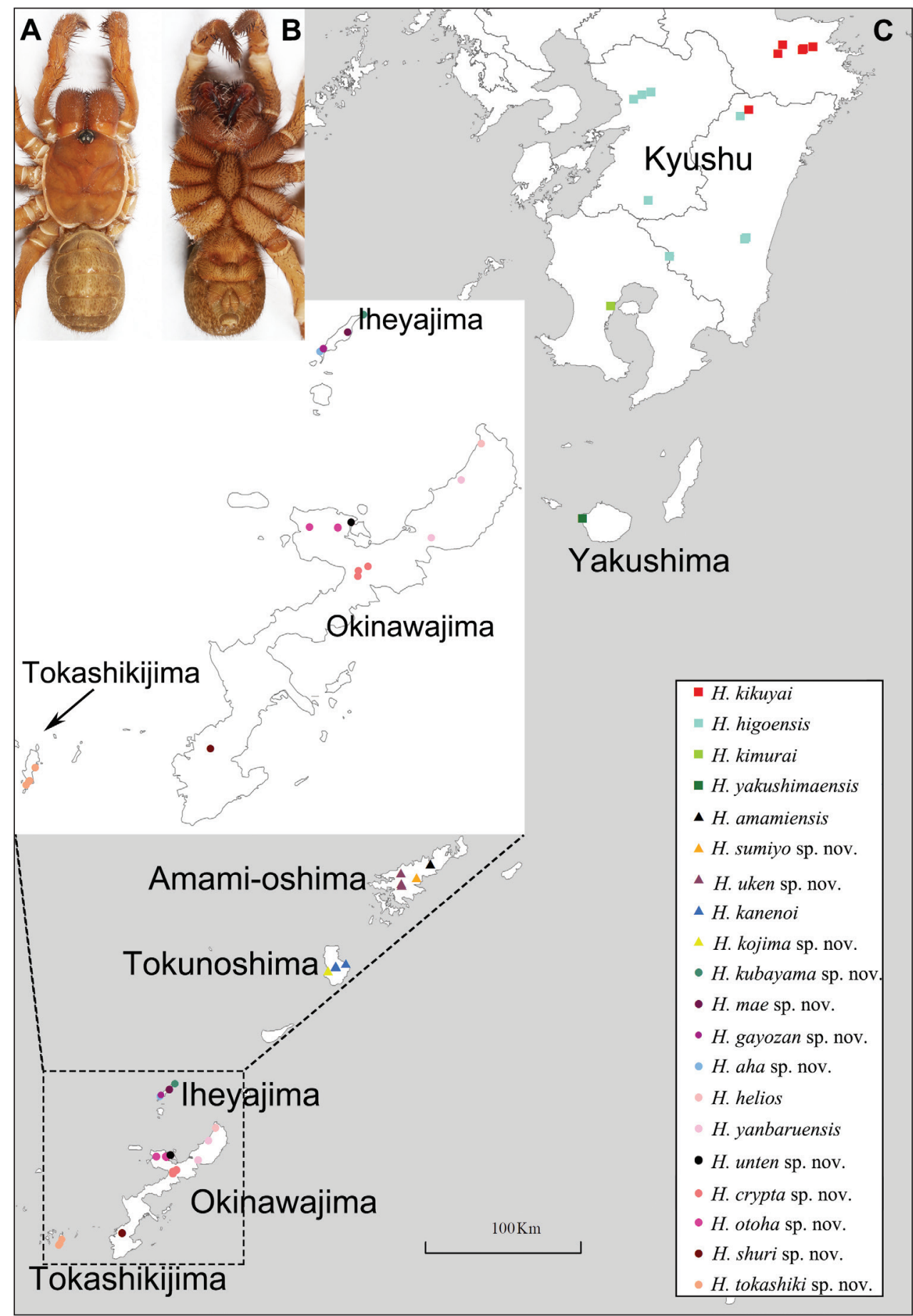

Figure I. General somatic morphology of Heptathela kimurai (Kishida, 1920) A dorsal view B ventral view and map showing the sampling localities of Heptathela specimens from Kyushu to central Ryukyus (C). 
Chinese, Vietnamese and some Japanese species (not Ryuthela) were within Heptathela sensu lato, and the names Nanthela and Sinothela became its synonyms. All the above hypotheses were based exclusively on morphology. Using combined molecular and morphological data, we recently showed Heptathela sensu lato to be paraphyletic, and redefined the genus to comprise only the species from Kyushu and from the Ryukyus (Xu et al. 2015a, b).

Heptathela therefore does not occur in continental Asia (Xu et al. 2015b); instead, its species are endemic to Kyushu (six known species) as well as the northern and central islands of the Ryukyu archipelago (Tanikawa and Miyashita 2014; World Spider Catalog 2019), two are known from Amamioshima and Tokunoshima, and another two from Okinawajima. As emphasised in the above overview, most known Heptathela species have been delimited solely morphologically. The study by Tanikawa and Miyashita (2014) is an exception as they discovered one species, $H$. helios Tanikawa $\&$ Miyashita, 2014, and delimited $H$. yanbaruensis Haupt, 1983 and $H$. helios from Okinawa using genetic distances. Our recent molecular data used to test biogeographical hypotheses within East Asian margins, on the other hand, suggested that over ten Heptathela species exist on these islands (Xu et al. 2016).

We follow this evidence with a thorough taxonomic revision of Heptathela. As is the case in other liphistiids, Heptathela females usually lack clear morphologically diagnostic characters and furthermore exhibit considerable intraspecific variation. While males are more readily diagnosable morphologically, they are very rarely collected (Haupt 2003; Schwendinger and Ono 2011; Tanikawa 2013; Tanikawa and Miyashita 2014; $\mathrm{Xu}$ et al. 2015c, 2017). A purely morphological revision would thus be preliminary, or even erroneous, and be an underestimation of true species diversity (Xu et al. 2017). Therefore, an integrative taxonomic revision of this genus is a preferred alternative. We first establish species hypotheses for all available Heptathela specimens using morphological diagnoses. We then use the evidence from a range of molecular species delimitation analyses from a parallel study (Xu et al. 2019) to test and further diagnose these species, and discuss the benefits of such taxonomic approaches.

\section{Materials and methods}

\section{Specimen acquisition}

Our original sampling, performed in the entire Heptathela range from Kyushu to the central Ryukyu archipelago, relied on the type locality information of each known Heptathela species, but also involved previously unexplored areas, focusing particularly on roadside habitats (Figs 1C, 2; for details of all specimens used in this study see Suppl. material 1: Table S1). We collected all specimens alive for initial check of their maturity status. We then fixed mature spiders in ethanol but retained all subadults alive to be reared to maturation. All mature specimens were subsequently preserved in $80 \%$ ethanol after being removed the right set of legs for the cryo-collection. 


\section{Morphological examination}

Specimens were morphologically examined under an Olympus SZX16 stereomicroscope and an Olympus BX51 compound microscope. Their genitalia were cleared in boiling $10 \% \mathrm{KOH}$ for a few minutes to dissolve soft tissues. Unless noted otherwise, left palps were imaged. All measurements are reported in millimetres. Leg and palp measurements are given in the following order: total length (femur + patella + tibia + metatarsus + tarsus). The value ranges for cheliceral groove denticles, spinnerets and measurements in taxonomic descriptions are based on all the examined specimens.

Taxonomic descriptions using morphological characteristics follow our established methodology (Xu et al. 2015c, 2017). We use the following abbreviations throughout:
ALE anterior lateral eyes,
E embolus,
AME anterior median eyes,
OL opisthosoma length,
BL body length,
OW opisthosoma width,
CL carapace length,
PC paracymbium,
Co conductor,
PLE posterior lateral eyes,
CT contrategulum,
PME posterior median eyes,
CW carapace width,
RC receptacular cluster,
D depression,
T tegulum.

Museum abbreviations:

CBEE Centre for Behavioural Ecology and Evolution, School of Life Sciences, Hubei University, Wuhan, China;

NMNS National Museum of Nature and Science, Japan;

NZMC National Zoological Museum of China, Institute of Zoology, Chinese Academy of Sciences, Beijing, China;

MCZ Museum of Comparative Zoology, Harvard University, Cambridge, USA;

ZMH Zoological Museum Hamburg, Germany.

Voucher specimens are deposited at CBEE, and the type specimens will be deposited in NMNS and NZMC.

\section{Integrative taxonomic framework}

The integrative taxonomic framework considers diagnosable lineages as potentially valid species. Our taxonomic approach is based on morphological diagnoses that provide an initial species hypothesis (19 species). This is then further tested within a parallel, molecular species delimitation study (Xu et al. 2019). The parallel study amplified two molecular loci, the classical animal barcoding region (COI) as well as the nuclear ITS2 for most sampled specimens, and analysed those data using a multi-tier species 


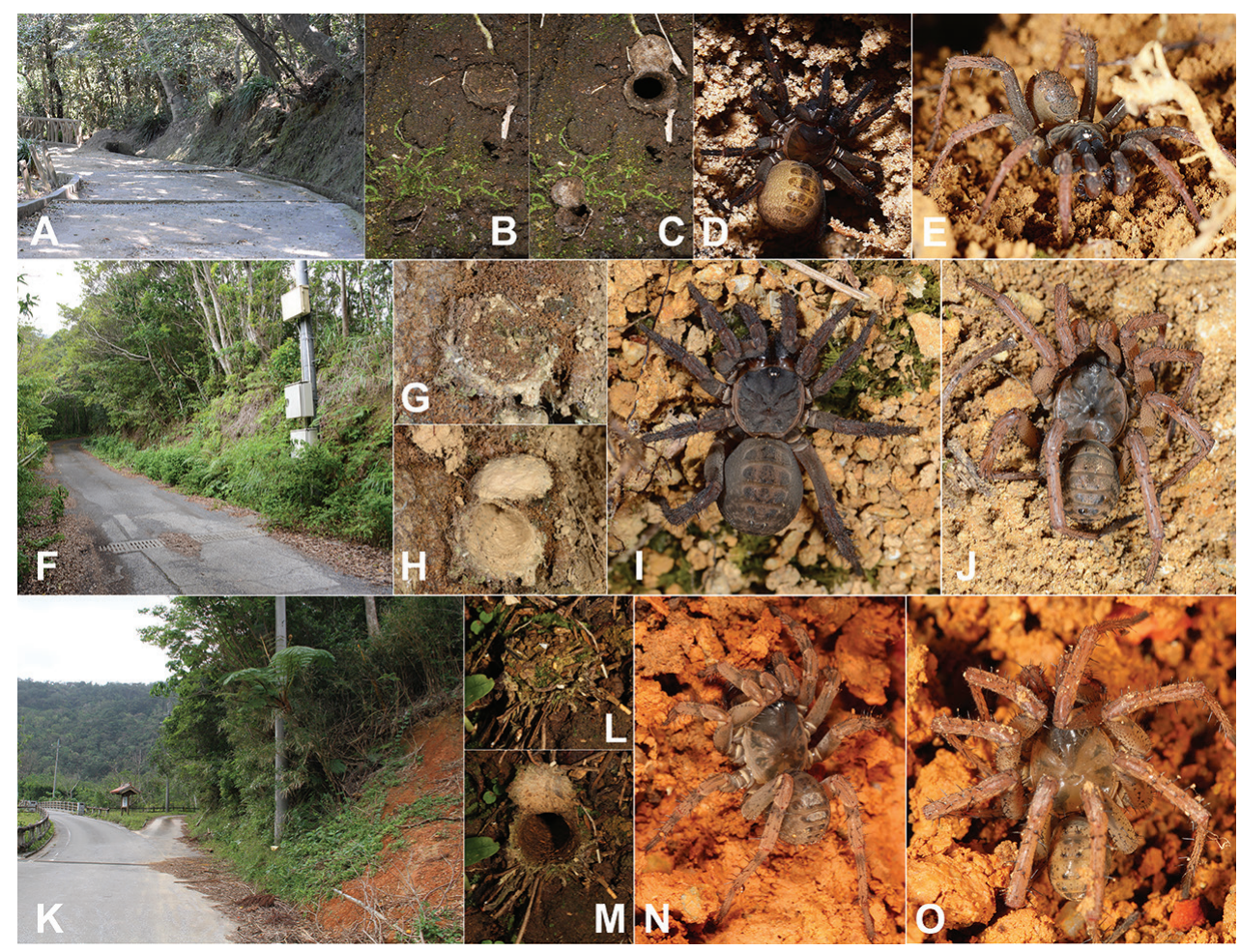

Figure 2. Microhabitats, trapdoors, and general somatic morphology of Heptathela Kishida, 1923 A-E Kyushu group: A microhabitat of $H$. kimurai (Kishida, 1920) at Shiroyama Park, Kyushu B, C open and closed trapdoor of $H$. kimurai $\mathbf{D}$ female $H$. kimurai E male $H$. higoensis (Haupt, 1983) F-J Amami group: $\mathbf{F}$ microhabitat of $H$. sumiyo sp. nov. at Sumiyo-cho, Amamioshima $\mathbf{G}, \mathbf{H}$ open and closed trapdoor of $H$. amamiensis Haupt, 1983 I female $H$. sumiyo sp. nov. J male $H$. sumiyo sp. nov. K-O Okinawa group: K microhabitat of $H$. yanbaruensis Haupt, 1983 at Yona, Okinawajima L, $\mathbf{M}$ open and closed trapdoor of $H$. yanbaruensis $\mathbf{N}$ female $H$. yanbaruensis $\mathbf{O}$ male $H$. yanbaruensis.

delimitation strategy. In tier 1 analysis, six different sequence-based species delimitation methods using the full data matrix containing 180 samples are used to test the here proposed number of morphologically delimited species of Heptathela. The fully congruent species supported by all the methods are included within the final species counts, but only the conflicting species enter the tier 2 analysis in which more genetic markers are added for the samples that represent those conflicting lineages and multilocus coalescent-based delimitation methods are used to test competing species models derived from tier 1 analysis. If conflict persists, our integrative approach re-evaluates DNA barcode gaps to delimit the competing species models derived from tier 2 analysis. The additional molecular diagnostic evidence, species specific nucleotide substitution information in the animal barcoding gene region (i.e. the standard alignment of COI) (Brower 2010; Cook et al. 2010; Planas and Ribera 2015), augments the morphological species diagnoses that in isolation would be prone to error.

The aligned DNA barcode gene COI is deposited in the Dryad Digital Repository. 


\section{Results and discussion}

Of the total species diversity of 20 , eight previously described species remain valid, two junior synonyms are proposed, and 12 new Heptathela species are discovered and described. This taxonomic revision thus represents a $137.5 \%$ increase in species diversity of the genus, drawing parallels with prior species richness underestimation found also in other liphistiid genera, e.g., Ganthela (Xu et al. 2015c) and Ryuthela (Xu et al. 2017).

To ease identification and to hint at putative evolutionary units, we divide Heptathela into the Kyushu, the Amami, and the Okinawa groups, each of which contains four, five, and ten species, respectively (Fig. 3). This division of groups is mostly based on species' morphology and geographical distributions, but also heeds the phylogeny. Hence, to avoid paraphyly of Okinawa group, we do not assign $H$. helios from northern Okinawajima to a group as it is sister to all the other Heptathela species (Fig. 3).

\section{Taxonomy of Heptathela}

\section{Genus Heptathela Kishida, 1923}

Type species. Liphistius kimurai Kishida, 1920: 235.

Diagnosis. Heptathela males differ from Liphistius males by lacking the tibial apophysis, and from males of all other Heptathelinae genera by possessing a leaf-shaped conductor (Fig. 4E, F, H), a wide and thumb-shaped embolus (Fig. 4F, I, K), and a wide tegulum with rugose margin (Fig. 4J, K). Heptathela females differ from Liphistius females by paired receptacular clusters, and from females of all other Heptathelinae genera by paired depressions on the ventro-lateral part of the genital atrium (Fig. 4C, $\mathrm{D})$, and by the paired receptacular clusters with the main and secondary, lateral, irregular receptacular clusters (Fig. 4A-D).

General description. All the 20 species share the following characteristics: sternum narrow, longer than wide; a few short pointed hairs running over ocular mound in a longitudinal row; chelicerae robust with promargin of cheliceral groove with variable sized denticles (the number of denticles is presented under each species description); legs with strong hairs and spines, and male legs distinctly longer than female legs; opisthosoma with 12 tergites, and the fifth tergite is the largest in all the species except in $H$. amamiensis, $H$. sumiyo sp. nov., and $H$. uken sp. nov., in which the fourth tergite is the largest.

\section{The Kyushu group}

Diagnosis. The males of the Kyushu group differ from those of the other two groups by the nearly rectangular contrategulum whose two proximal thirds are serrated (Fig. $4 \mathrm{H}$, I), whereas it is semi-elliptic in the others. The Kyushu group females resemble those 


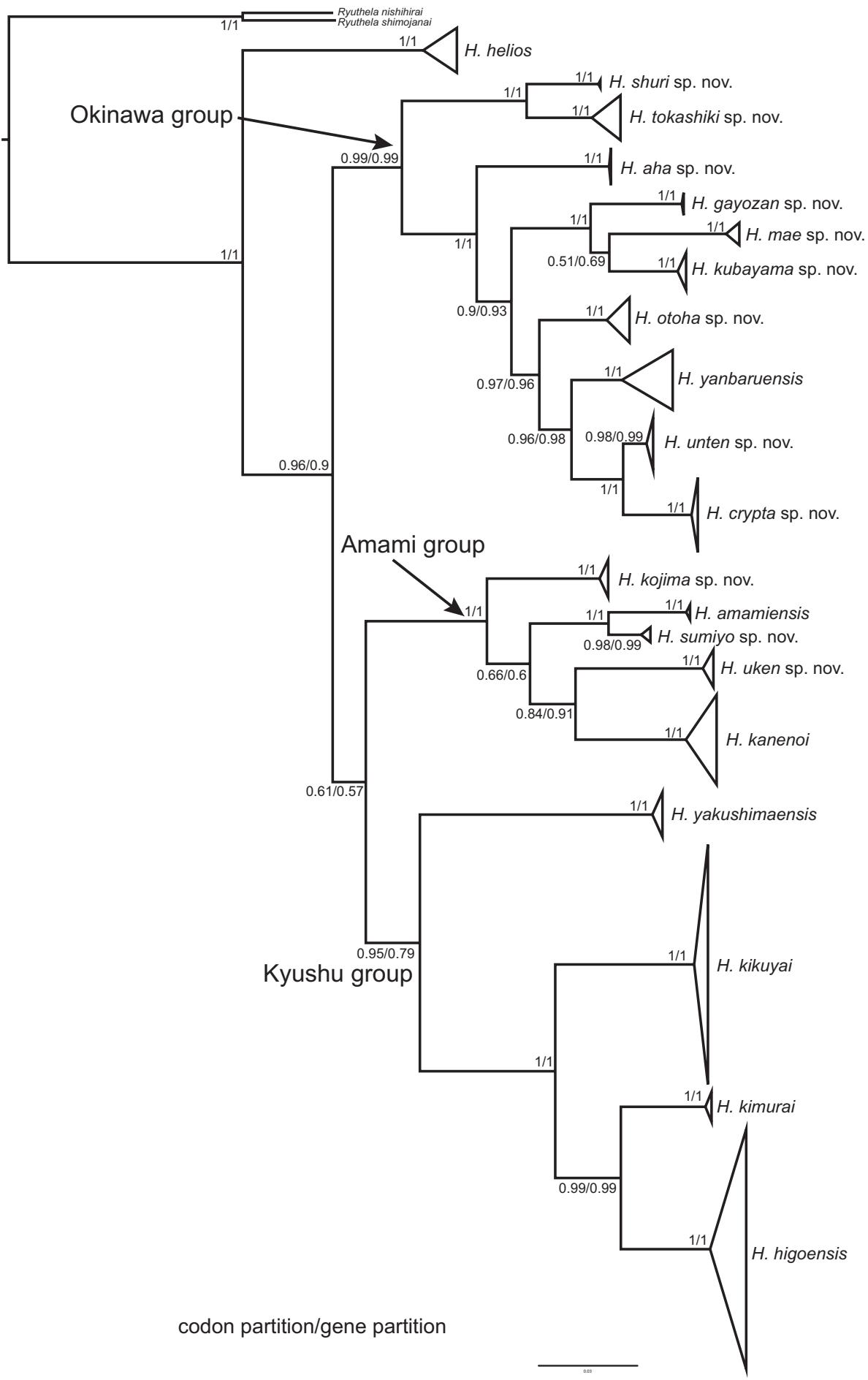

Figure 3. A simplified species-level phylogeny derived from the BI tree in the parallel paper (Xu et al. 2019) showing three species groups. 
of the Okinawa group, but differ from those of the Amami group by distinctly paired depressions on the ventro-lateral part of the genital atrium (Fig. 4C, D).

Monophyly. The Bayesian analyses based on concatenated two genes and two partitions (for details, see Xu et al. 2019) inferred the same topology, which supports the Kyushu group monophyly (posterior probability, hereafter PP = 0.95/0.79) (Fig. 3).

Composition. H. higoensis Haupt, 1983, H. kikuyai Ono, 1998, H. kimurai (Kishida, 1920), and H. yakushimaensis Ono, 1998.

Distribution. Kyushu and Yakishima (Fig. 1C).

\section{Heptathela higoensis Haupt, 1983}

Figs 4, 5

Heptathela kimurai higoensis Haupt, 1983: 283 (holotype: male, from Kumamoto, Kyushu, Japan, collected by M. Yoshikura on 27 September 1973, deposited in MCZ, examined); Haupt 2003: 69. Heptathela higoensis: Ono 1998: 16; Ono 2009: 80; Ono and Ogata 2018: 26, 479.

Heptathela nishikawai Ono, 1998: 19 (holotype: female, from Hitoyoshi-shi, Kumamoto-ken, Kyushu, Japan, collected by H. Ono on 19 November 1996, deposited in NMNS, examined); Ono 2009: 83; syn. nov.

Heptathela yaginumai Ono, 1998: 20 (holotype: female, from Honjo, Kunitomi-cho, Higashimorokata-gun, Miyazaki-ken, Kyushu, Japan, collected by T. Yaginuma on 18 June 1949, deposited in NMNS, examined); Ono 2009: 81. syn. nov.

Diagnosis. Males of $H$. higoensis can be distinguished from those of $H$. kikuyai by one of the embolus peaks being longer than the other (Fig. $4 \mathrm{H}, \mathrm{I}, \mathrm{K}$ ) and by the slightly blunt tegular marginal apophysis (Fig. 4J), and from those of H. yakushimaensis by the conductor with the weakly serrated prolateral margin (Fig. 4F, H, K). Females of $H$. higoensis can be distinguished from those of $H$. kimurai by the wide and flat dorso-posterior part of the genital area (Fig. 4A, B), and from those of $H$. kikuyai and $H$. yakushimaensis by the inner receptacular clusters that are larger than the outer ones (Fig. 4A-D). Moreover, H. higoensis differs from all other Kyushu group Heptathela species by the following unique nucleotide substitutions in the standard DNA barcode alignment: G (140), C (146), A (179), C (251), C (257), C (263), G (272), A (326), C (332), T (350), G (479), G (569), C (572), A (578), G (596), G (632), G (641).

Description. Males $(N=11)$. Carapace yellow brown; opisthosoma light brown, with dark brown tergites close to each other; cheliceral groove with 11-13 denticles; 7 or 8 spinnerets. Measurements: BL 8.80-11.00, CL 4.40-5.25, CW 4.10-4.90, OL 4.40-5.60, OW 2.90-3.40; ALE > PLE > PME > AME; leg I 11.75 $(3.45+1.50+2.60+2.70+1.50)$, leg II $12.40(3.40+1.60+2.50+3.10+1.80)$, leg III $13.30(3.20+1.60+2.40+3.80+2.30)$, leg IV $16.80(4.40+1.20+3.30$ $+5.20+2.70)$. 


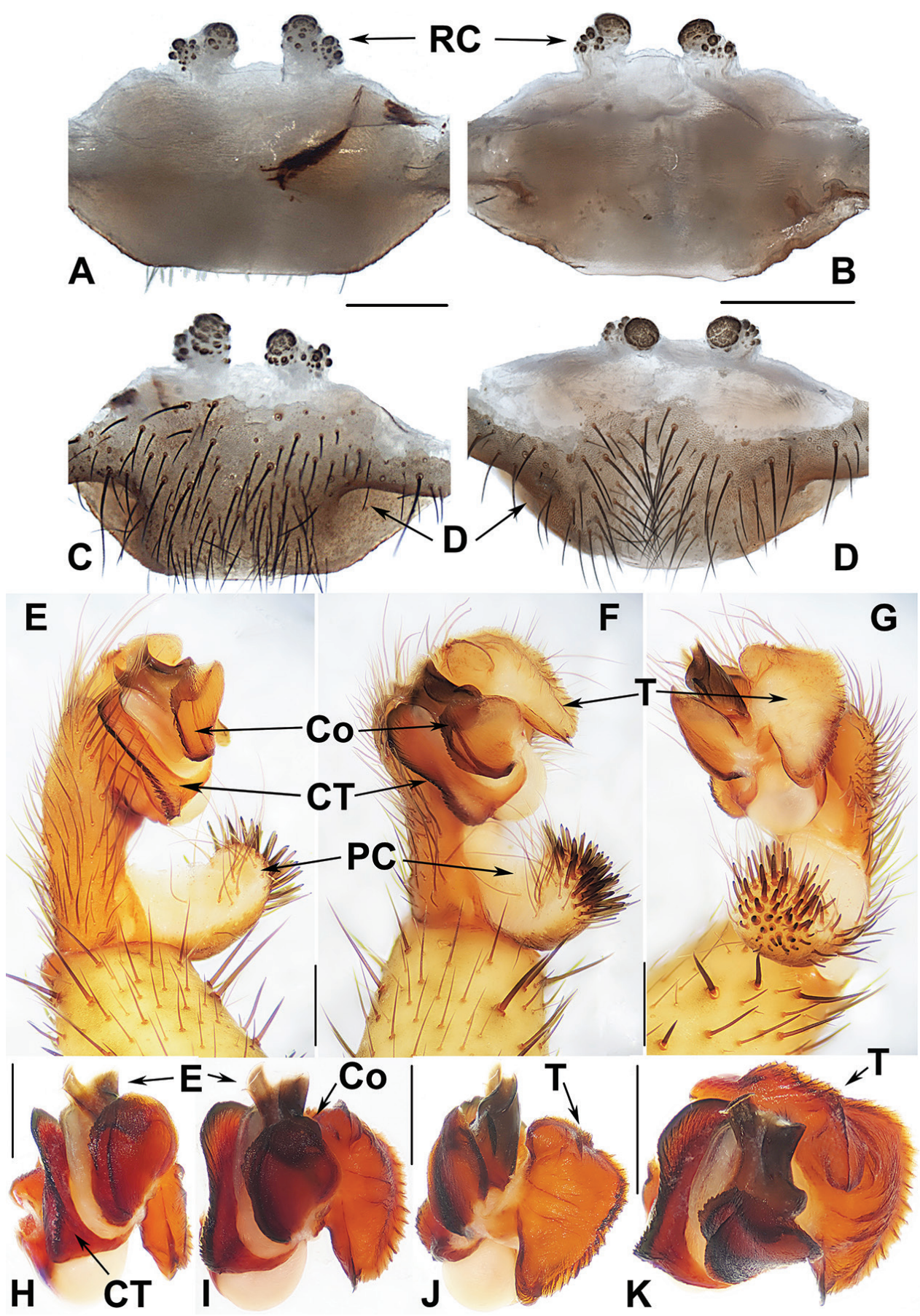

Figure 4. Male and female genital anatomy of Heptathela higoensis Haupt, 1983 A, C 3472 (short for XUX-2013-472) B, D 3435 E-G 3365 H-K 3381 A, B vulva dorsal view C, D vulva ventral view E palp prolateral view $\mathbf{F}$ palp ventral view $\mathbf{G}$ palp retrolateral view $\mathbf{H}-\mathbf{K}$ palp distal view; 3365: Hitoyoshi Ruins Park, Kumamoto; 3472: Takachihokawara, Kagoshima; 3435: Mukoyama, Miyazaki; 3381: Kozomo, Kumamoto. Scale bar: $0.5 \mathrm{~mm}$. 

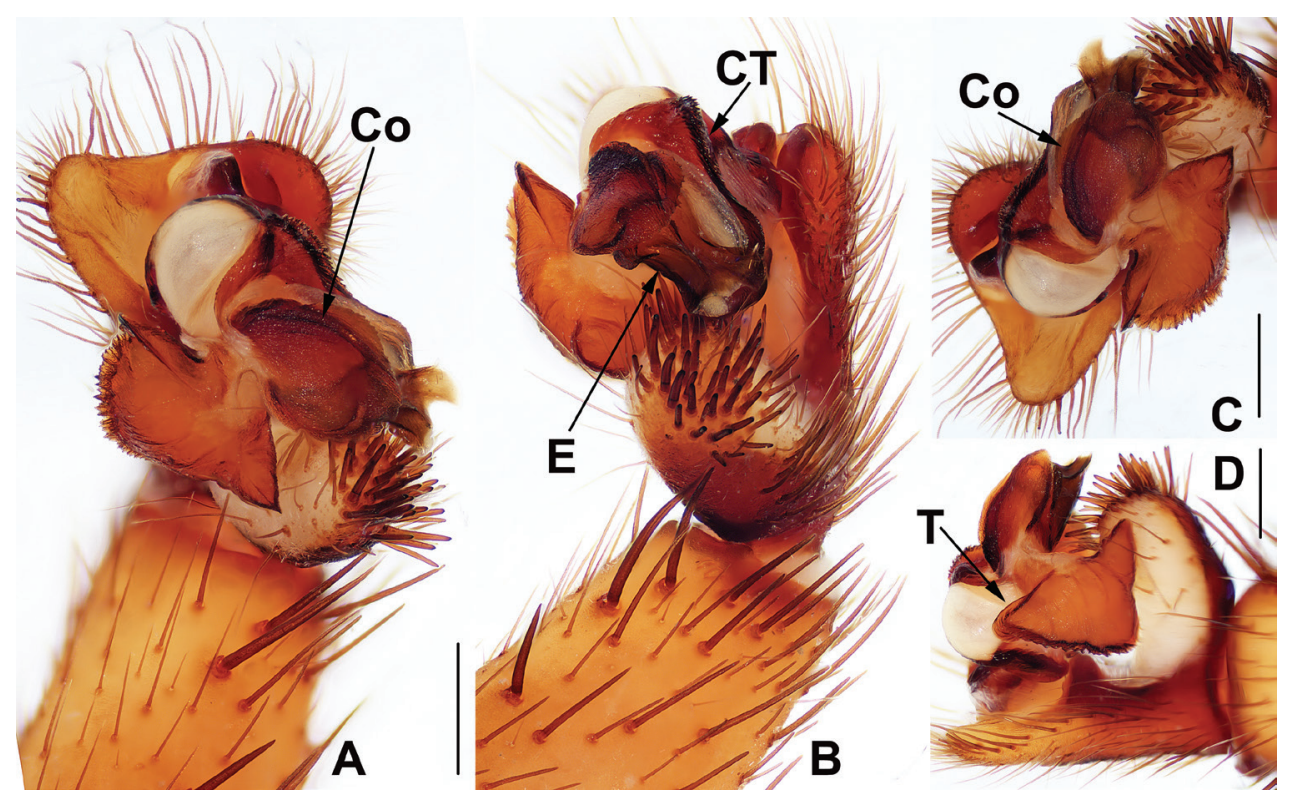

Figure 5. Male holotype genital anatomy of Heptathela higoensis Haupt, 1983 (MCZ 21751) A palp ventral view B palp retrolateral view C, D palp distal view. Scale bar: $0.5 \mathrm{~mm}$.

Palp. Prolateral side of paracymbium unpigmented and unsclerotised, numerous setae and spines at the tip of paracymbium (Fig. 4E-G). Contrategulum with serrated margin proximally and smooth margin distally (Fig. 4I, K). Tegulum wide, the dorsal extension of terminal apophysis and marginal apophysis with a serrated margin retrolaterally (Fig. 4J, K). Conductor wide and with an apical tooth and a deep fold (Fig. $4 \mathrm{H}, \mathrm{K}$ ). Embolus with two peaks, one peak longer than the other, and with a curved margin retrolaterally (Fig. 4H-K).

Females $(N=43)$. Carapace and opisthosoma colour as in male; cheliceral groove with 11-16 pronounced denticles; tergites similar to male; 7-8 spinnerets. Measurements: BL 8.00-12.80, CL 4.30-6.10, CW 3.80-5.57, OL 4.10-6.50, OW 2.70-4.90; ALE $>$ PLE > PME > AME; palp $8.20(2.70+1.50+1.70+2.30)$, leg I $8.80(2.80+$ $1.75+1.70+1.55+1.00)$, leg II $8.93(2.75+1.60+1.55+1.83+1.20)$, leg III 9.30 $(2.70+1.60+1.50+2.20+1.30)$, leg IV $13.25(3.65+1.80+2.40+3.40+2.00)$.

Female genitalia. A pair of depressions on the ventro-lateral part of genital atrium (Fig. 4C, D). Paired receptacular clusters along the anterior margin of bursa copulatrix, divided into two parts, the inner main part forming a large granulate tubercle, with short genital stalks, the outers with several small granules (Fig. 4).

Remarks. We examined the male holotype of H. higoensis (Fig. 5) and identified the species as $H$. higoensis even though the bulb of holotype male is relatively distorted compared to the specimens we collected. After we examined 11 males and 43 females collected at the type localities of $H$. higoensis, $H$. nishikawai and $H$. yaginumai, and compared the holotypes of $H$. higoensis, $H$. nishikawai and $H$. yaginumai with our specimens, we proposed synonymy of $H$. nishikawai and $H$. yaginumai with 
$H$. higoensis based on their genital morphology, molecular species delimitation $(\mathrm{Xu}$ et al. 2019), and intraspecific genetic distances, $0-1.19 \%$ (K2P) and $0-1.18 \%$ ( $p$ distance) among 43 specimens, although females exhibit a considerable intraspecific variation in genitalia.

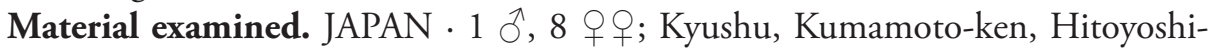
shi, Fumotomachi, Hitoyoshi Ruins Park; 32.21N, 130.77E; alt. 140 m; 18 September 2013; D. Li and B. Wu leg.; XUX-2013-365 (ô matured 19 July 2014 at

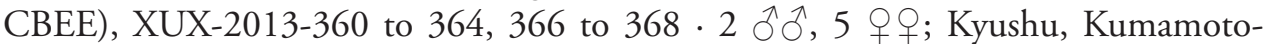
ken, Kumamoto-shi, Tatsutayama, Tatsuta National Park; 32.82N, 130.73E; alt. 60

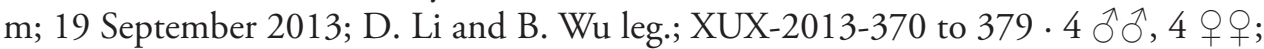
Kyushu, Kumamoto-ken, Kumamoto-shi, Higashi-ku, Kozono 1-chome; 32.84N, 130.78E; alt. 100 m; 19 September 2013; D. Li and B. Wu leg.; XUX-2013-380 to 389 (XUX-2013-381, $\widehat{\jmath}$ matured 2 August 2014 at CBEE) - 1 ઈ, 3 q 9 ; Kyushu, Kumamoto-ken, Kumamoto-shi, Kasuga, Hanaokayama; 32.80N, 130.68E; alt. 120 m, 19 September 2013; D. Li and B. Wu leg.; XUX-2013-390 to 393 . 3 우; Miyazaki-ken, Nishiusuki-gun, Takachiho-cho, Mukoyama; 32.70N, 131.30E; alt. 320 m; 22 September 2013; D. Li and B. Wu leg.; XUX-2013-435 to $441 \cdot 1$ §, 3 우; Miyazaki-ken, Higashimorokata-gun, Kunitomi-cho, Honjo 11960-1; 32.00N, 131.34E; alt. 30 m; 23 September 2013; D. Li and B. Wu leg.; XUX-2013-456 (ð,, matured 19 July 2014 at CBEE), XUX-2013-449 to $451 \cdot 1$ ภ, 12 우; Miyazaki-ken, Higashimorokata-gun, Kunitomi-cho, Honjo 4191; 31.98N, 131.33E; alt. 30 m; 23 September 2013; D. Li and B. Wu leg.; XUX-2013-457 to 467C • 1 q; Miyazaki-ken, Nishimorokata-gun, Takaharu-cho, Kamamuto, Lake Miike; 31.89N, 130.96E; alt. 360 m; 23 September 2013; D. Li and B. Wu leg.; XUX-2013-468 • 1 ô, 4 qo+; 2 Kagoshima-ken, Kirishima-shi, Kirishima, Takachihokawara; 31.89N, 130.89E; alt.

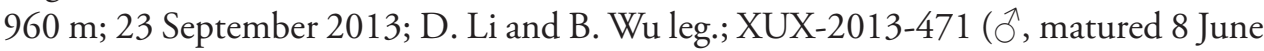
2014 at CBEE), XUX-2013-472 to 474, 476.

Distribution. The species is known from the following prefectures on the Japanese island Kyushu: Kumamoto-ken (Hitoyoshi-shi and Kumamoto-shi), Miyazaki-ken (Nishiusuki-gun, Higashimorokata-gun and Nishimorokata-gun), Kagoshima-ken (Kirishima-shi) (Fig. 1C).

\section{Heptathela kikuyai Ono, 1998}

Fig. 6

Heptathela kikuyai Ono, 1998: 16 (holotype: male, from Mt. Gozaga-dake, 20 km south of Oita-shi, Oita-ken, Kyushu, Japan, collected by N. Kikuya on 13 September 1979, deposited in NMNS, examined); Ono 2009: 81; Schwendinger and Ono 2011: 613; Ono and Ogata 2018: 26, 479.

Diagnosis. Males of $H$. kikuyai differ from those of $H$. higoensis by the two embolus peaks of a similar height, and the hooked tegular marginal apophysis (Fig. 6J, K), and from those of $H$. yakushimaensis by only a weakly serrated prolateral margin of the con- 

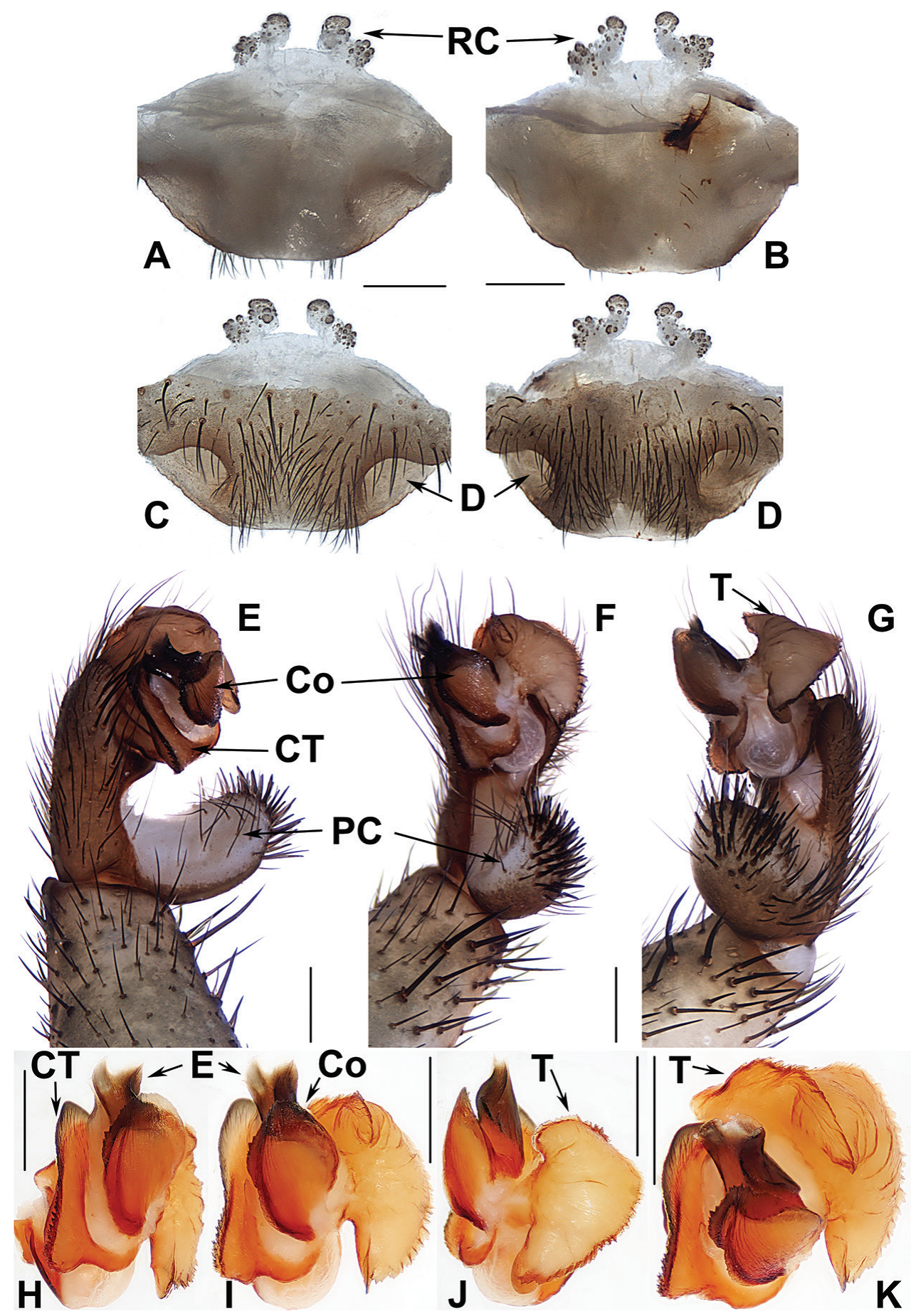

Figure 6. Male and female genital anatomy of Heptathela kikuyai Ono, 1998 A, C 3409 (short for XUX-2013-409) B, D 3401 E-K 3405 A, B vulva dorsal view C, D vulva ventral view E palp prolateral view $\mathbf{F}$ palp ventral view $\mathbf{G}$ palp retrolateral view $\mathbf{H}-\mathbf{K}$ palp distal view; 3405, 3409: Mt. Gozaga-dake, Oita-ken; 3401: Onomachi, Oita-ken. Scale bar: 0.5 mm. 
ductor (Fig. 6H, I). Females of H. kikuyai differ from those of all other Kyushu group Heptathela species by the inner receptacular clusters with longer and slender stalks (Fig. 6A-D). Moreover, H. kikuyai differs from all other Kyushu group Heptathela species by the following unique nucleotide substitutions in the standard DNA barcode alignment: G (11), A (33), C (41), C (59), C (68), A (83), A (89), C (116), T (194), A (212), T (222), A (239), A (251), C (260), T (276), T (287), C (305), C (329), A (345), T (365), C (470), G (491), T (546), T (578), T (581), G (635), C (656).

Description. Males $(N=5)$. Carapace and opisthosoma brown, with dark-spotted tergites; cheliceral groove with 10-12 denticles; 6 or 7 spinnerets. Measurements: BL 7.90-9.40, CL 4.00-5.05, CW 3.55-4.30, OL 3.90-4.60, OW 2.60-3.20; ALE > PLE > PME > AME; leg I $13.65(3.90+1.90+2.75+3.30+1.80)$, leg II $13.35(3.45$ $+1.60+2.65+3.55+2.10)$, leg III $14.40(3.30+1.50+2.75+4.45+2.40)$, leg IV $19.25(4.85+1.90+3.35+6.00+3.15)$.

Palp. Prolateral side of paracymbium unpigmented and unsclerotised, numerous setae and spines at the tip of paracymbium (Fig. 6E-G). Contrategulum with serrated margin proximally and smooth margin distally (Fig. 6H, I, K). Tegulum wide, the dorsal extension of terminal apophysis and hooked marginal apophysis of tegulum with a serrated margin retrolaterally (Fig. 6J, K). Conductor wide and with an apical tooth and a deep fold (Fig. 6I, K). Embolus with two peaks at the slightly same level and a curved margin retrolaterally (Fig. $6 \mathrm{H}-\mathrm{K}$ ).

Females $(N=30)$. Carapace and opisthosoma as in male; cheliceral groove with 12-17 pronounced denticles; tergites similar to male; $6-8$ spinnerets. Measurements: BL 8.55-15.10, CL 4.30-7.90, CW 3.80-6.70, OL 4.40-8.75, OW 2.80-6.10; ALE $>$ PLE $>$ PME > AME; palp $9.15(3.25+1.60+1.90+2.40)$, leg I $10.30(3.30+1.90$ $+1.90+2.00+1.20)$, leg II $10.15(3.10+1.80+1.85+2.10+1.30)$, leg III 10.80 $(3.00+1.80+1.80+2.60+1.60)$, leg IV $15.80(4.50+2.20+2.80+4.10+2.20)$.

Female genitalia. A pair of depressions on the ventro-lateral part of genital atrium. Paired receptacular clusters along the anterior margin of bursa copulatrix, indistinctly divided into two parts, with many small granules, inner ones with longer and slender genital stalks (Fig. 6A-D).

Material examined. JAPAN - 6 우; Oita-ken, Bungoono-shi, Totoki, Onomachi; 33.06N, 131.52E; alt. 240 m; 20 September 2013; D. Li and B. Wu leg.; XUX-2013394 to 396, 399 to $401 \cdot 2$ 수, 4 우웅 Oita-ken, Bungoono-shi, Onomachi-Ando, Mt. Gozaga-dake; 33.11N, 131.55E; alt. 630 m; 20 September 2013; D. Li and B. Wu leg.; XUX-2013-404, 405, 408, 409, 411, 412 (405, ô matured 2 May 2014 at CBEE) $\cdot 2$ ○े 6 , 6 + ; Oita-ken, Usuki-shi, Takeyama; 33.10N, 131.72E; alt. 80 m; 20 September 2013; D. Li and B. Wu leg.; XUX-2013-414, 416 to 422 - 1 \%, 8 우우; Oita-ken, Usuki-shi, Inukai-machi Sasamuta; 33.09N, 131.67E; alt. 120 m; 21 September 2013; D. Li and B. Wu leg.; XUX-2013-424 to 430, 432, 433 - 6 우우 Miyazaki-ken, Nishiusuki-gun, Takachiho-cho, Iwato; 32.73N, 131.35E; alt. 400 m; 22 September 2013; D. Li and B. Wu leg.; XUX-2013-442 to 444, 446 to 448.

Distribution. The species is known from the following prefectures on the Japanese island Kyushu: Oita-ken (Bungoono-shi and Usuki-shi) and Miyazaki-ken (Nishiusuki-gun) (Fig. 1C). 


\section{Heptathela kimurai (Kishida, 1920)}

Fig. 7

Liphistius kimurai Kishida, 1920: 362 (holotype: female, from Shiroyama, Kagoshima, Kyushu, Japan, collected by A. Kimura in October 1920, lost in the Science College Museum of the Tokyo Imperial University (Haupt, 1983); neotype: male, from the same locality as for the original type specimen, collected by J. Haupt on 21 March 1982, matured in August 1982, deposited in ZMH, but the neotype may be lost according to Dunlop et al. 2014).

Heptathela kimurai Kishida, 1923: 236; Bristowe 1933: 1030; Sawaguti and Ozi 1937: 116 (partly); Yaginuma 1954: 15; Yaginuma 1955: 35; Yaginuma 1960: 19; Yaginuma 1971: 19; Gertsch and Platnick 1979: 5; Yaginuma 1979: 1; Yaginuma 1980: 44; Haupt 1983: 283; Yoshikura 1983: 63; Haupt 1984: 163; Yaginuma 1986: 1; Yoshikura 1987: 148; Chikuni 1989b: 18; Ono 1998: 14; Yoo and Kim2002: 27; Haupt 2003: 69; Ono 2009: 81; Schwendinger and Ono 2011: 614; Ono and Ogata 2018: 25, 479 .

Diagnosis. Females of $H$. kimurai resemble $H$. higoensis females but differ by a slightly curved dorso-posterior margin of the genital area (Fig. 7A, B). Moreover, H. kimurai differs from all other Kyushu group Heptathela species by the following unique nucleotide substitutions in the standard DNA barcode alignment: C (26), T (98), G (191), G (194), G (206), C (215), T (251), T (278), G (293), C (362), T (366), G (443), T (449), C (452), G (506), G (521), C (527), G (548), T (572), C (599), A (615), C (638).

Description. Females $(N=9)$. Carapace yellow brown; opisthosoma brown, with brown tergites close to each other; cheliceral groove with 12-14 vestigial denticles; seven spinnerets. Measurements: BL 9.30-13.80, CL 4.88-6.30, CW 4.10-5.50, OL 4.80-7.30, OW 3.45-5.40; ALE > PLE > PME > AME; palp $7.77(2.57+1.50+1.60$ $+2.10)$, leg I $9.15(2.85+1.70+1.75+1.65+1.20)$, leg II $8.88(2.65+1.65+1.55$ $+1.88+1.15)$, leg III $9.63(2.70+1.70+1.60+2.30+1.33)$, leg IV $14.08(3.88+$ $1.90+2.50+3.60+2.20)$.

Female genitalia. A pair of depressions on the ventro-lateral part of genital atrium. Paired of receptacular clusters along the anterior margin of bursa copulatrix, divided into two parts, the inner main part forming a large granulate tubercle, the outer part with several small granules (Fig. 7A-D).

Male: unknown.

Remarks. We could not examine the presumably lost neotype male (Dunlop et al. 2014). The male is therefore unknown. The non-topotypical male described as $H$. kimurai by Ono $(1998,2009)$ is excluded here as we are not sure that the specimen was collected from the type locality although it was obtained in Kagoshima-shi.

Material examined. JAPAN . 9 q ; Kagoshima-ken, Kagoshima-shi, Shiroyamacho, Shiroyama Park; 31.60N, 130.55E; alt. 100 m; 18 September 2013; D. Li and B. Wu leg.; XUX-2013-349, 351, 352, 354 to 359.

Distribution. The species is known from the Kagoshima prefecture on the Japanese island Kyushu (Fig. 1C). 


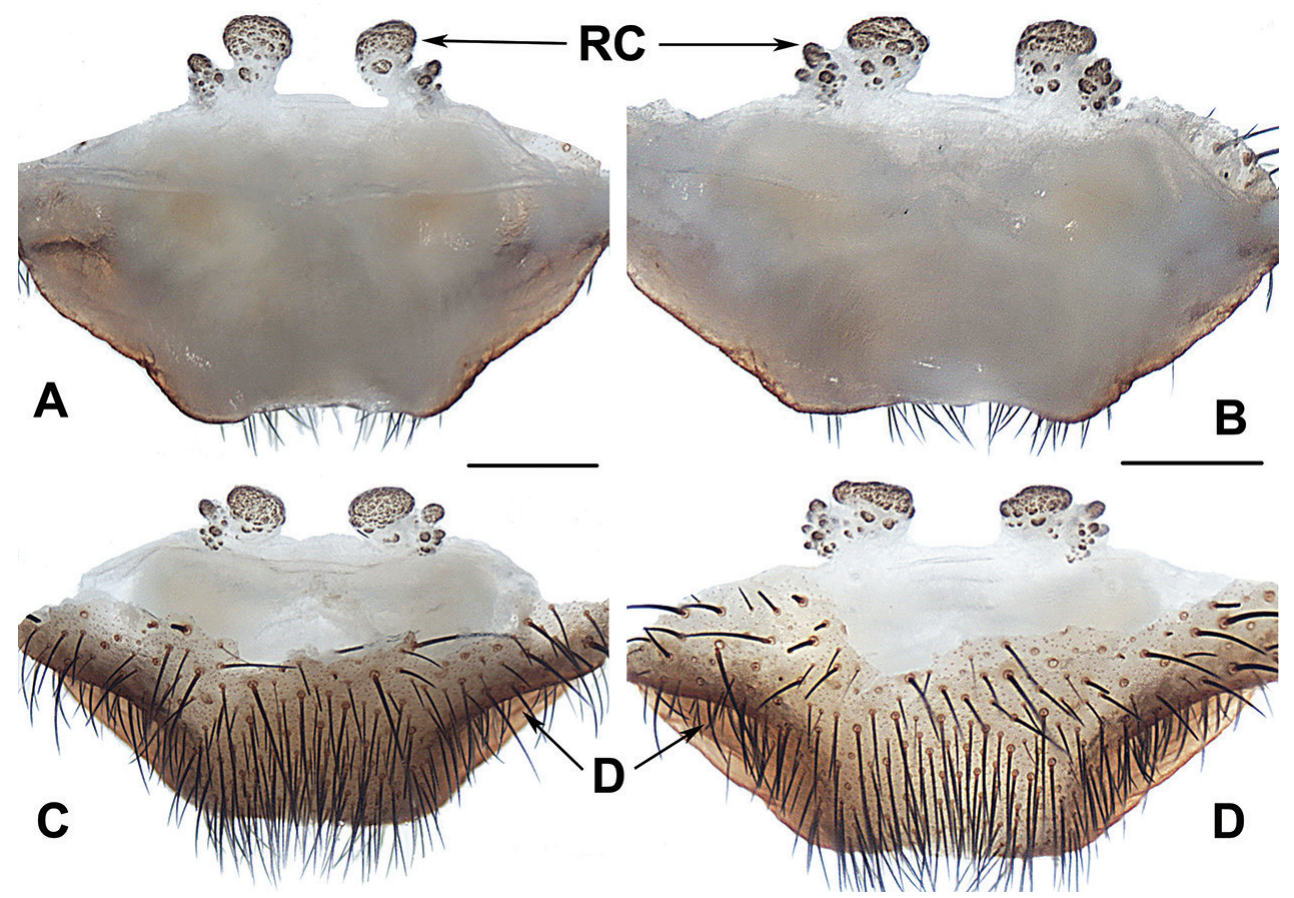

Figure 7. Female genital anatomy of Heptathela kimurai (Kishida, 1920) A, C 3351 (short for XUX2013-351) B, D 3359 A, B vulva dorsal view C, D vulva ventral view. Scale bar: $0.5 \mathrm{~mm}$.

\section{Heptathela yakushimaensis Ono, 1998}

Fig. 8

Heptathela yakushimaensis Ono, 1998: 23 (holotype: female, from Mt. Kunibaidake, Yakushima Island, Kagoshima-ken, Japan, collected by A. Tanikawa on 15 July 1990, deposited in NMNS, examined); Ono 2009: 83.

Heptathela kimurai yakushimaensis: Haupt 2003: 69.

Diagnosis. Males of H. yakushimaensis differ from those of all other Kyushu group Heptathela species by a strongly serrated prolateral conductor margin (Fig. 8G, H, J, K), the tapered tegular marginal apophysis (Fig. 8L, M), and a larger tegular terminal apophysis (Fig. 8L, M). Females of $H$. yakushimaensis differ from those of $H$. kimurai and $H$. higoensis by the finely granulated inner receptacular clusters that are smaller than the outer ones (Fig. 8A-F), and from those of $H$. kikuyai by the inner receptacular clusters lacking well defined stalks. H. yakushimaensis also differs from all other Kyushu group Heptathela species by the following unique nucleotide substitutions in the standard DNA barcode alignment: T (56), A (68), T (74), G (77), C (84), C (89), C (95), G (98), C (107), A (110), C (122), T (131), T (143), T (164), T (167), C (188), C (200), C (212), T (215), C (216), G (218), T (236), G (242), T (248), G (251), C (278), A (284), T (293), C (294), C (308), T (323), C (347), C (356), T (392), T (395), C (396), T (401), T (407), 


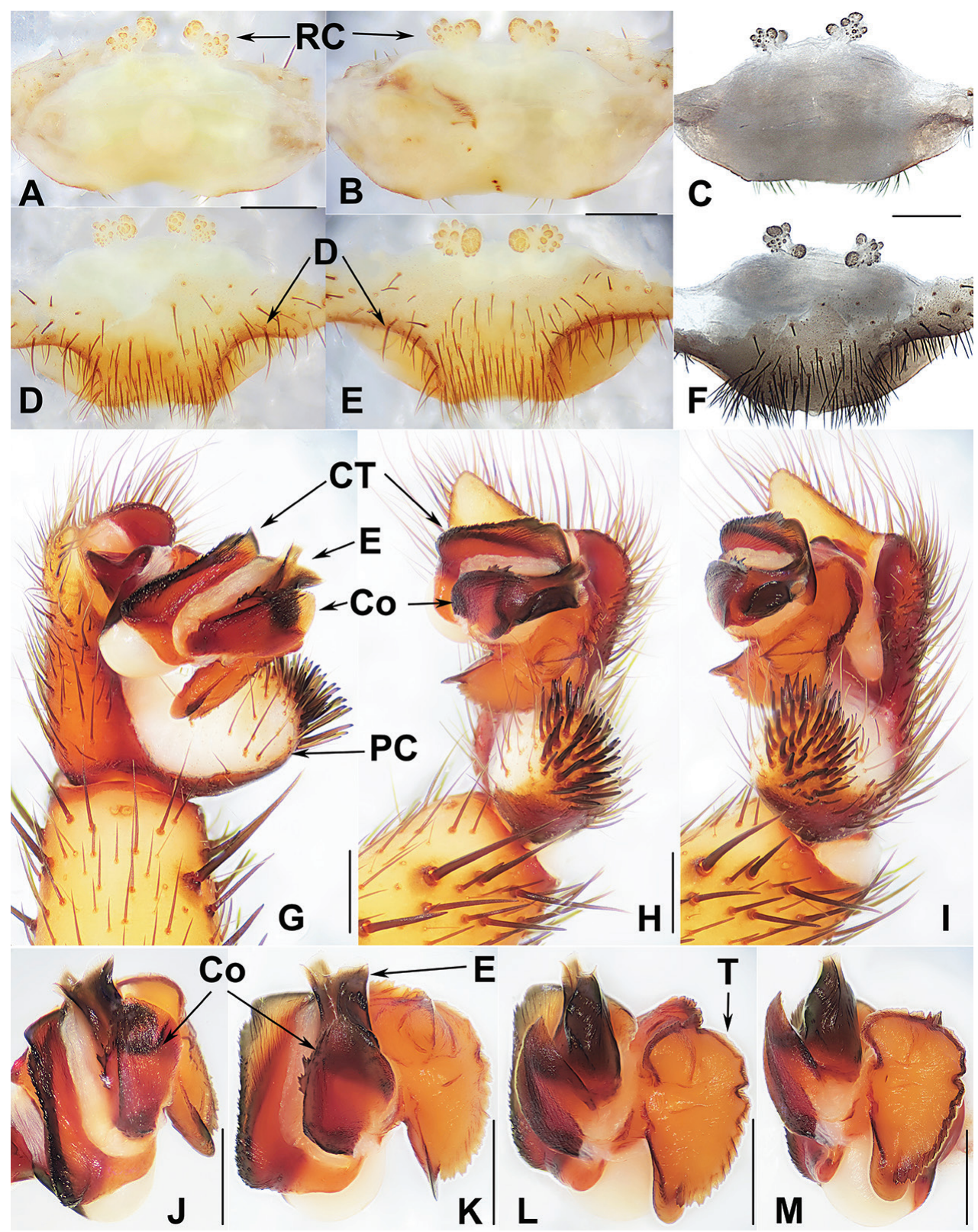

Figure 8. Male and female genital anatomy of Heptathela yakushimaensis Ono, 1998 A, D 3491 (short for XUX-2013-481) B, E 3495 C, F 3494 G-M 3500 A-C vulva dorsal view D-F vulva ventral view $\mathbf{G}$ palp prolateral view $\mathbf{H}$ palp ventral view I palp retrolateral view J-M palp distal view. Scale bar: $0.5 \mathrm{~mm}$.

C (411), C (413), C (417), G (422), G (425), C (437), C (438), A (443), T (455), T (458), T (461), T (482), T (488), C (510), T (528), A (530), A (536), A (539), T (548), G (557), C (567), C (584), T (591), T (632), T (635), G (638), T (650), A (665). 
Description. Males $(N=2)$. Carapace brown; opisthosoma light brown, with dark brown tergites close to each other; cheliceral groove with 10-13 denticles; 7 spinnerets. Measurements: BL 8.70-10.50, CL 4.27-5.50, CW 4.00-4.90, OL 3.80-6.10, OW 2.40-4.40; ALE > PLE > PME > AME; leg I $13.27(3.70+1.60+2.72+3.50+1.75)$, leg II $13.71(3.55+1.65+2.80+3.75+1.96)$, leg III $14.65(3.52+1.65+2.78+$ $4.50+2.20)$, leg IV $18.64(4.35+1.70+3.72+6.20+2.67)$.

Palp. Prolateral side of paracymbium unpigmented and unsclerotised, numerous setae and spines at the tip of paracymbium (Fig. 8G-I). Contrategulum with serrated margin proximally and smooth margin distally, and slightly curved at the proximal $2 / 3$ of contrategulum (Fig. 8G, H, J, K). Tegulum with serrated margin, widest in the middle (Fig. 8K-M). Conductor prolateral margin strongly serrated (Fig. 8G, H, J, K). Embolus wide with two peaks (Fig. 8G-K).

Females $(N=8)$. Carapace and opisthosoma colour as in male; cheliceral groove with $12-15$ pronounced denticles; tergites similar to male; 7 spinnerets. Measurements: BL 9.10-14.80, CL 4.50-6.80, CW 4.05-5.90, OL 4.70-8.60, OW 3.006.40; ALE > PLE > PME > AME; palp $9.72(3.22+1.70+2.10+2.70)$, leg I 11.15 $(3.45+1.90+2.05+2.35+1.40), \operatorname{leg}$ II $9.61(3.07+1.90+1.00+2.36+1.28)$, leg III $11.75(3.25+1.95+2.05+2.80+1.70)$, leg IV $16.30(4.25+2.25+2.90$ $+4.40+2.50)$.

Female genitalia. A pair of depressions on the ventro-lateral part of genital atrium. A pair of receptacular clusters along the anterior margin of bursa copulatrix, divided into two parts, the inner part is similar or smaller than the outer part, on which there are several small granules (Fig. 8A-F).

Material examined. JAPAN · $2 \hat{\jmath}, 8$ + $O$; Kagoshima-ken, Kumage-gun, Yakushima-cho, Mt. Kankake-dake; 30.37N, 130.39E; alt. 170 m; 24 September 2013; D. Li and B. Wu leg.; XUX-2013-490, 491, 493 to 500 (500, o matured 2 August 2014 at CBEE).

Distribution. The species is known from the Kagoshima prefecture on the Japanese island Yakushima (Fig. 1C).

\section{The Amami group}

Diagnosis. The males of the Amami group differ from those of the other two groups by the rugose conductor with a spiniform apex, the contrategulum with a strongly serrated margin whereas it is nearly rectangular in Kyushu group and weakly serrated in Okinawa group, and the embolus with a wide and flat opening (Fig. 9I-L). The females of the Amami group differ from those of the other two groups by indistinctly paired depressions on the ventro-lateral part of the genital atrium and the inner receptacular clusters without tubercula (Fig. 9A-E).

Monophyly. The Bayesian analyses based on concatenated two genes and two partitions (for details, see $\mathrm{Xu}$ et al. 2019) inferred the same topology, supporting the Amami group monophyly ( $\mathrm{PP}=1 / 1)$ (Fig. 3). 
Composition. H. amamiensis Haupt, 1983, H. kanenoi Ono, 1996, H. kojima sp. nov., $H$. sumiyo sp. nov., and $H$. uken sp. nov.

Distribution. Amamioshima and Tokunoshima (Fig. 1C).

\section{Heptathela amamiensis Haupt, 1983}

Fig. 9

Heptathela kimurai amamiensis Haupt, 1983: 283 (holotype: female, from Naze, Amami-oshima, Japan, collected by J. Haupt on 26 March 1980, deposited in $\mathrm{ZMH}$, holotype presumably lost (Dunlop et al. 2014); Haupt 2003: 69. Heptathela amamiensis: Ono and Nishikawa 1989: 120; Ono 2009: 80; Ono and Ogata 2018: 27, 479 .

Diagnosis. Males of $H$. amamiensis differ from those of $H$. sumiyo sp. nov. by the wider saddle-shaped embolus in the prolateral view, and the narrower conductor base in the ventral view (Fig. 9G, J-L); from those of $H$. kanenoi and H. kojima sp. nov. by the spiniform conductor apex (Fig. 9F, I, J). Females of $H$. amamiensis resemble those of other Amami group Heptathela species but can be distinguished from those of $H$. kanenoi by the tuberculate outer receptacular clusters (Fig. 9B-E). H. amamiensis can also be diagnosed from all other Amami group Heptathela species by the following unique nucleotide substitutions in the standard DNA barcode alignment: C (89), A (179), A (194), T (215), T (218), C (273), A (281), C (284), A (327), G (332), G (362), C (467), C (543), C (647).

Description. Male. Carapace brown; opisthosoma light brown, with dark brown tergites; cheliceral groove with 13 denticles; 8 spinnerets. Measurements: BL 12.85, CL 6.50, CW 5.98, OL 6.65, OW 4.00; ALE > PLE > PME > AME; leg I $18.25(5.00+$ $2.45+3.80+4.60+2.40), \operatorname{leg}$ II $18.75(4.85+2.40+3.75+5.00+2.75), \operatorname{leg}$ III 20.10 $(4.90+2.60+3.60+5.90+3.10)$, leg IV $25.30(6.00+2.60+5.10+7.70+3.90)$.

Palp. Prolateral side of paracymbium unpigmented and unsclerotised, numerous setae and spines at the tip of paracymbium (Fig. 9F-H). Contrategulum with serrated margin (Fig. 9F, G, I, J). Tegulum wide, with dentate dorsal extension of terminal apophysis (Fig. 9H, L) and blunt terminal apophysis (Fig. 9H, K, L). Conductor sclerotised and rugose, with several folds and a spiniform apex (Fig. 9F-L). Embolus sclerotised, with a wide and flat opening, the distal part slightly sclerotised, and saddleshaped in the prolateral view (Fig. 9F-L).

Females $(N=5)$. Carapace and opisthosoma colour as in male; cheliceral groove with 12-14 pronounced denticles; tergites similar to those of male; seven or eight spinnerets. Measurements: BL 11.08-16.90, CL 5.45-6.90, CW 4.70-6.20, OL 6.619.10, OW 4.70-7.38; ALE > PLE > PME > AME; palp $12.36(4.12+2.11+2.75+$ 3.38), leg I $14.12(4.52+2.50+2.70+2.83+1.57)$, leg II $14.06(4.25+2.37+2.61$ $+3.15+1.68)$, leg III $15.13(4.27+2.56+2.55+3.55+2.20), \operatorname{leg}$ IV $21.86(5.84+$ $2.91+4.08+5.81+3.22)$. 


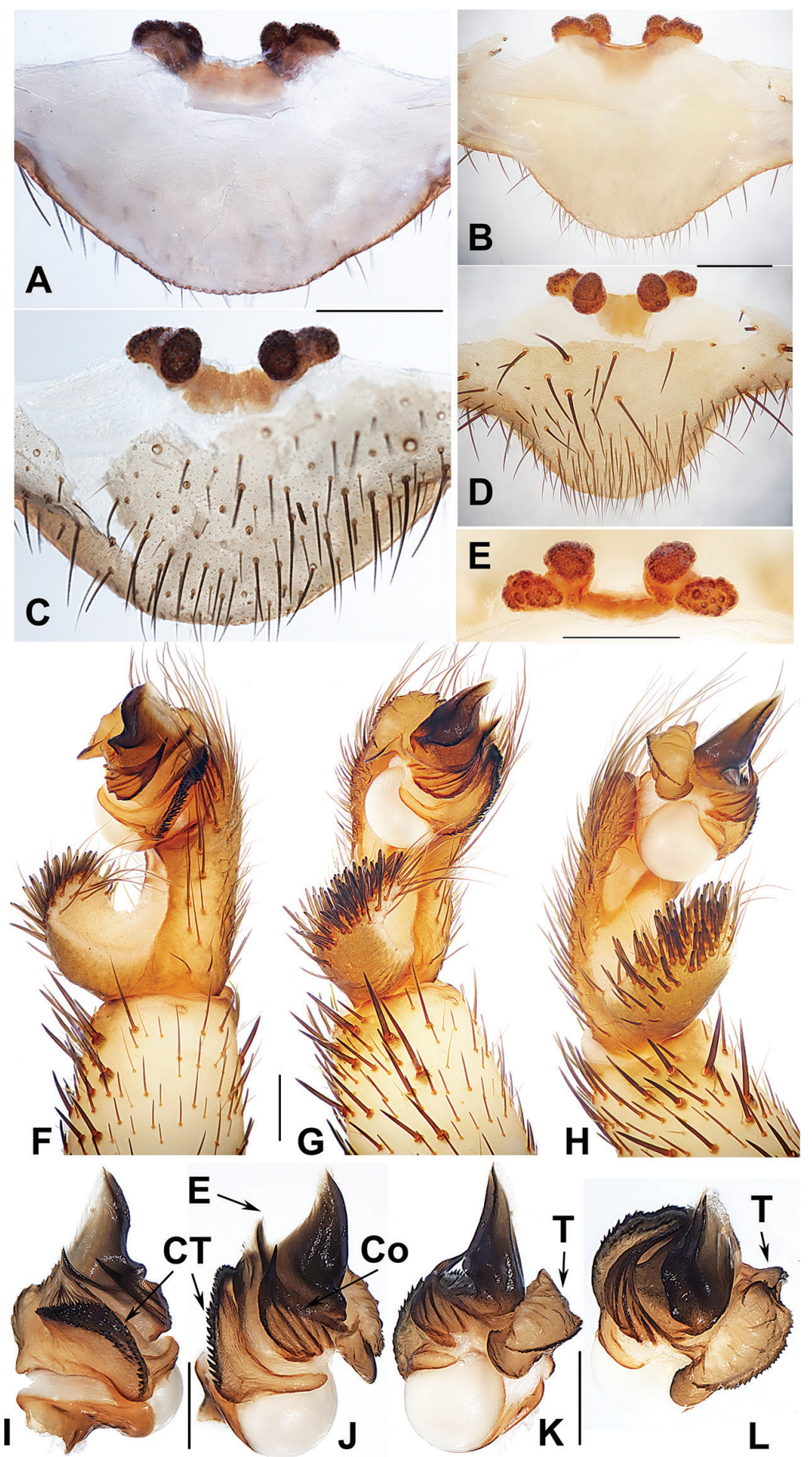

Figure 9. Male and female genital anatomy of Heptathela amamiensis Haupt, 1983 A, C 3278 (short for XUX-2013-278) B, D, E 3285 F-L 3283 A, B vulva dorsal view C, D vulva ventral view E vulva distal view $\mathbf{F}$ right palp prolateral view $\mathbf{G}$ right palp ventral view $\mathbf{H}$ right palp retrolateral view $\mathbf{I}-\mathbf{L}$ left palp distal view. Scale bar: $0.5 \mathrm{~mm}$. 
Female genitalia. A pair of indistinct depressions on the ventro-lateral part of genital atrium (Fig. 9C, D). Paired receptacular clusters along the anterior margin of bursa copulatrix, divided into two parts, the inners ovate, the outers tuberculate, without genital stalks (Fig. 9A-E).

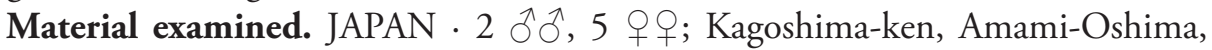
Amami-shi, Nazehirata-cho, Michinoshima Loop Bridge; 28.36N, 129.50E; alt. 60 m; 15 September 2013; D. Li and B. Wu leg.; XUX-2013-276, 278, 281 to 285.

Distribution. The species is known from the Japanese island Amamioshima (Fig. 1C).

\section{Heptathela kanenoi Ono, 1996}

Fig. 10

Heptathela kanenoi Ono, 1996: 158 (holotype: male, from Mikyo, Amagi-cho, Tokunoshima, Kagoshima-ken, Japan, collected by M. Owada and S. Kaneno on 2 November 1992, deposited in NMNS, examined); Ono 2009: 80.

Diagnosis. Males of $H$. kanenoi can be distinguished from those of all other Amami group Heptathela species by lacking a spiniform conductor apex (Fig. 10G, I). Females of H. kanenoi can be distinguished from those of all other Amami group Heptathela species by the inner receptacular clusters larger than the outers (Fig. 10B, C). H. kanenoi can also be diagnosed from all other Amami group Heptathela species by the following unique nucleotide substitutions in the standard DNA barcode alignment: A (35), G (38), C (71), C (125), C (224), G (278), T (281), C (288), T (332), G (359), C (396), C (410), G (443), T (449), A (512), C (533), G (557), C (560), $\mathrm{C}$ (623), $\mathrm{T}$ (641).

Description. Males $(N=6)$. Carapace yellow brown; opisthosoma brown, with dark-spotted tergites close to each other; cheliceral groove with 10-13 vestigial denticles; 7 spinnerets. Measurements: BL 9.80-11.60, CL 5.10-6.00, CW 4.60-5.40, OL 5.15-5.60, OW 4.00-4.85; ALE > PLE > PME > AME; leg I $17.85(4.80+2.30$ $+3.80+4.60+2.35)$, leg II $18.90(4.90+2.30+3.80+5.20+2.70)$, leg III 20.80 $(4.70+2.30+4.00+6.50+3.30)$, leg IV $26.00(6.20+2.50+5.10+8.20+4.00)$.

Palp. Prolateral side of paracymbium unpigmented and unsclerotised, numerous setae and spines at the tip of paracymbium (Fig. 10D-F). Contrategulum with serrated margin (Fig. 10G-I). Tegulum with dentate dorsal extension of terminal apophysis (Fig. 10J) and blunt tegulum terminal apophysis (Fig. 10I, J). Conductor base wide and rugose, with several folds and each fold with an apical tooth (Fig. 10G-J). Embolus with a wide and flat opening, the distal part slightly sclerotised, and saddle-shaped in the prolateral view (Fig. 10H-I).

Females $(N=11)$. Carapace and opisthosoma colour as in male; cheliceral groove with 12-14 pronounced denticles; tergites similar to male; seven or eight spinnerets. Measurements: BL 8.30-12.90, CL 4.30-6.28, CW 3.60-5.40, OL 4.38-6.50, OW 3.70-6.20; ALE > PLE > PME > AME; palp $7.00(2.50+1.35+1.55+1.60)$, leg 

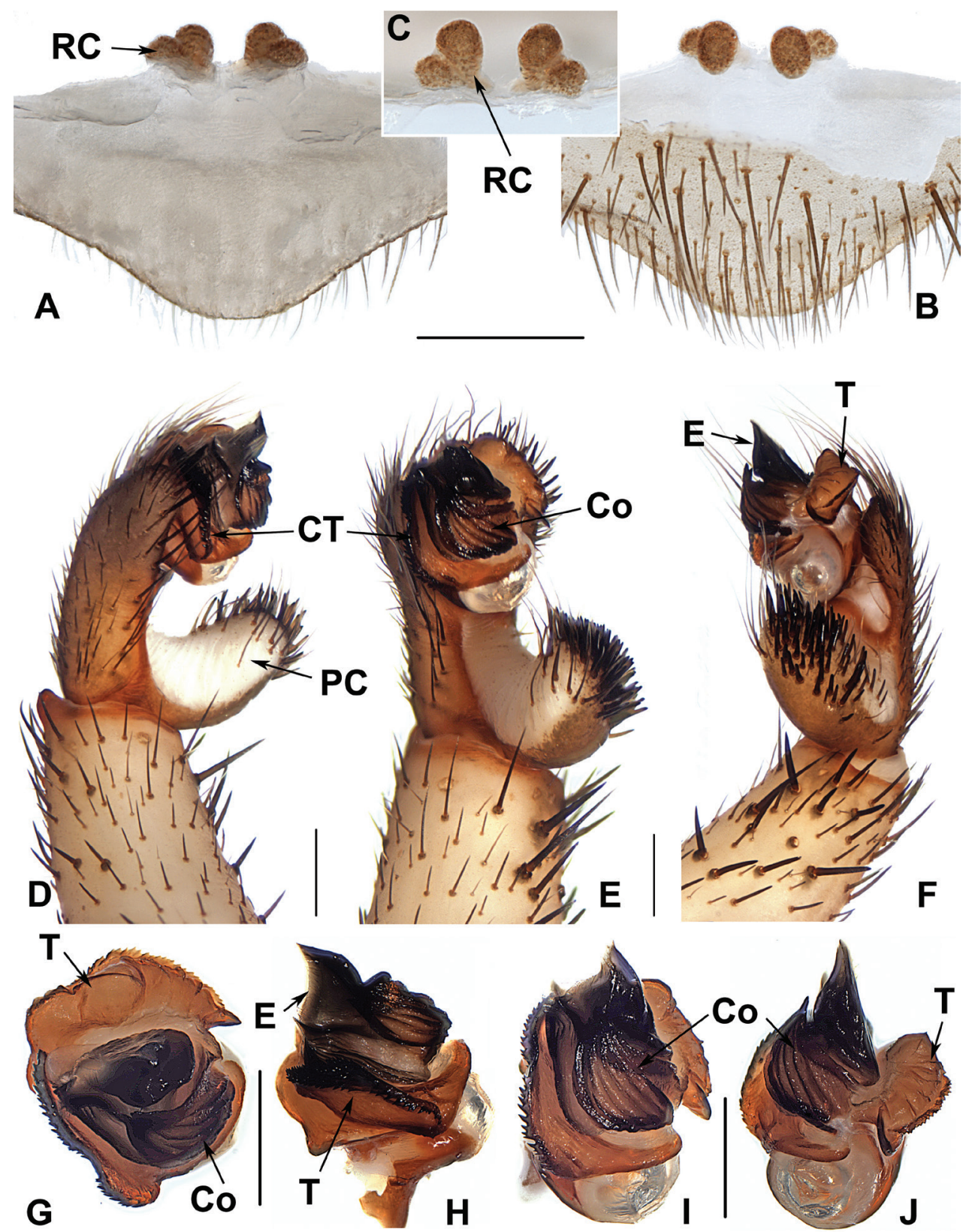

Figure 10. Male and female genital anatomy of Heptathela kanenoi Ono, 1996 A-C 3338 (short for XUX-2013-338) D-J 3334 A vulva dorsal view B vulva ventral view $\mathbf{C}$ vulva distal view $\mathbf{D}$ palp prolateral view $\mathbf{E}$ palp ventral view $\mathbf{F}$ palp retrolateral view $\mathbf{G}-\mathbf{J}$ palp distal view. Scale bar: $0.5 \mathrm{~mm}$.

I $7.75(2.30+1.25+1.60+1.50+1.10)$, leg II $7.60(2.60+1.10+1.50+1.50+$ $0.90)$, leg III $8.35(2.45+1.50+1.10+2.00+1.30)$, leg IV $12.15(3.40+1.50+$ $2.25+3.20+1.80)$. 
Female genitalia. A pair of indistinct depressions on the ventro-lateral part of genital atrium. Paired receptacular clusters along the anterior margin of bursa copulatrix, divided into two parts, the inners larger than the outers, without genital stalks (Fig. 10A-C).

Material examined. JAPAN $\cdot 1 \hat{\partial}, 1$; Kagoshima-ken, Tokunoshima, Amagicho, Mikyo; 27.77N, 128.95E; alt. 180 m; 16 September 2013; D. Li and B. Wu leg.;

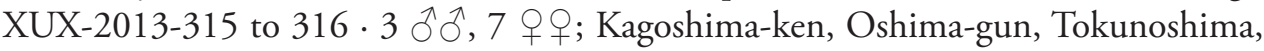
Tokunoshima-cho, Tokuwase; 27.79N, 129.01E; alt. 150 m; 17 September 2013; D.

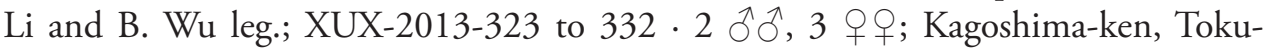
noshima, Amagi-cho, Mikyo; 27.77N, 128.95E; alt. 130 m; 17 September 2013; D. Li and B. Wu leg.; XUX-2013-333 to 338.

Distribution. The species is endemic to the Japanese island Tokunoshima (Fig. 1C).

\section{Heptathela kojima sp. nov.}

http://zoobank.org/9330F719-A1B4-4169-9FBF-90D13CD49248

Fig. 11

Type material. Holotype: JAPAN · つ̋'; Kagoshima-ken, Oshima-gun, Tokunoshima, Isen-cho, Kojima; 27.74N, 128.91E; alt. 160 m; 17 September 2013; D. Li and B. Wu leg.; XUX-2013-346 (matured 10 October 2013 at CBEE).

Paratypes: JAPAN $\cdot 2$ §ð, 6 우; same data as for holotype; XUX-2013-339, 340,342 to $345,347,348$.

Diagnosis. Males of $H$. kojima sp. nov. differ from those of $H$. amamiensis and $H$. kanenoi by a wide leaf-shaped conductor (Fig. 11I, J), and a less dentate dorsal extension of the tegular terminal apophysis (Fig. 11G, J, K), from those of $H$. sumiyo sp. nov. by a shallow saddle-shaped in the prolateral view, and from those of $H$. uken sp. nov. by embolus with two longer peaks (Fig. 11I, J). Females of H. kojima sp. nov. resemble those of other Amami group Heptathela species but differ from those of other Amami group Heptathela species by paired receptacular clusters close to each other (Fig. 11B, D). H. kojima sp. nov. can also be diagnosed from all other Amami group Heptathela species by the following unique nucleotide substitutions in the standard DNA barcode alignment: C (44), C (56), C (128), A (131), C (134), C (137), C (155), G (158), G (176), T (230), T (245), C (269), T (320), C (357), C (377), A (378), A (443), C (446), G (464), A (479), C (518), G (521), T (554), A (560), C (608), C (611).

Description. Male (Holotype). Carapace brown; opisthosoma light brown, with dark brown tergites close to each other; cheliceral groove with eleven denticles; seven spinnerets. Measurements: BL 7.30, CL 3.60, CW 3.30, OL 3.60, OW 2.70; ALE > PLE > PME > AME; leg I $14.90(4.20+165+3.15+4.00+1.90)$, leg II $16.10(4.20$ $+1.85+3.25+4.40+2.40)$, leg III $16.85(4.25+1.90+3.20+5.00+2.50), \operatorname{leg}$ IV $21.70(5.50+2.00+4.20+6.90+3.10)$.

Palp. Prolateral side of paracymbium unpigmented and unsclerotised, numerous setae and spines at the tip of paracymbium (Fig. 11E-G). Contrategulum with serrated 

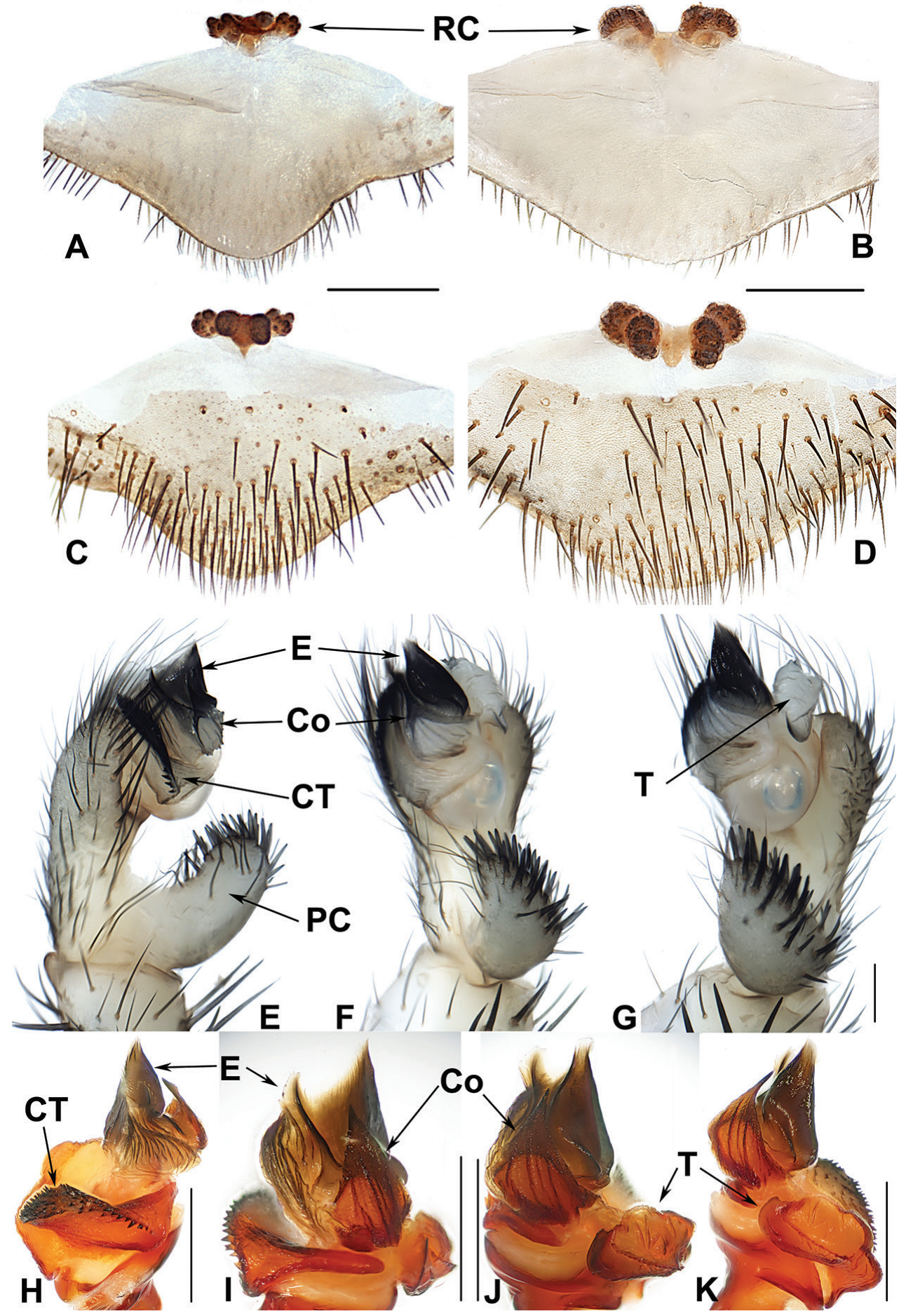

Figure I I. Male and female genital anatomy of Heptathela kojima sp. nov. A, C 3339 (short for XUX2013-339) B, D 3340 E-G 3346 (holotype) H-K 3344 A, B vulva dorsal view C, D vulva ventral view E palp prolateral view $\mathbf{F}$ palp ventral view $\mathbf{G}$ palp retrolateral view $\mathbf{H}-\mathbf{K}$ palp distal view. Scale bar: $0.5 \mathrm{~mm}$. 
margin (Fig. 11E, H, I). Conductor base wide, leaf-shaped and rugose, with several folds and gradually narrowing to a short spiniform apex (Fig. 11I, J). Embolus sclerotised, with a wide and flat opening (Fig. $11 \mathrm{H}-\mathrm{K}$ ).

Females $(N=6)$. Carapace and opisthosoma colour as in male; cheliceral groove with 13 pronounced denticles; tergites similar to male; 6-8 spinnerets. Measurements: BL 9.00-11.50, CL 4.90-6.15, CW 4.25-5.20, OL 4.30-6.10, OW 3.004.50; ALE > PLE > PME > AME; palp $9.05(3.25+1.65+1.85+2.30)$, leg I 10.50 $(3.35+1.80+2.00+2.10+1.25)$, leg II $10.05(3.10+1.80+1.75+2.10+1.30)$, leg III $10.45(2.90+1.95+1.65+2.40+1.55)$, leg IV $15.20(4.40+2.20+2.60$ $+4.00+2.00)$.

Female genitalia. A pair of indistinct depressions on the ventro-lateral part of genital atrium (Fig. 11C, D). Paired receptacular clusters separated from each other along the anterior margin of bursa copulatrix, or fused together, divided into two parts, without genital stalks (Fig. 11A-D).

Etymology. The species epithet, a noun in apposition, refers to the type locality.

Distribution. The species is endemic to the Japanese island Tokunoshima (Fig. 1C).

\section{Heptathela sumiyo sp. nov.}

http://zoobank.org/BB1A7494-C5CD-4D69-BC49-67052BCF7E05

Fig. 12

Type material. Holotype: JAPAN - ठ̋; Kagoshima-ken, Amami-Oshima, Amami-shi, Sumiyo-cho, Santaro-toge Pass; 28.28N, 129.42E; alt. 360 m; 15 September 2013; D. Li and B. Wu leg.; XUX-2013-293.

Paratypes: JAPAN · 4 ふึ, 6 우; same data as for holotype; XUX-2013-287 to 292, 294 to $296 \mathrm{~B}$.

Diagnosis. Males of $H$. sumiyo sp. nov. can be distinguished from those of $H$. $k a$ nenoi by the spiniform conductor apex (Fig. 12A, E, F), from those of $H$. amamiensis by the narrow and deeper saddle-shaped embolus in the prolateral view (Fig. 12A, E, F). Females of $H$. sumiyo sp. nov. resemble those of the other Amami group Heptathela species but differ from those of $H$. kaneno $i$ by the tuberculate lateral receptacular clusters that are equal in size, or slightly larger than the inner clusters (Fig. 12J, K). $H$. sumiyo sp. nov. also differs from all other Heptathela species of the Amami group by the following unique nucleotide substitutions in the standard DNA barcode alignment: $G$ (92), C (218), A (227), G (281), C (308), A (363), T (647).

Description. Male (Holotype). Carapace brown; opisthosoma light brown, with dark brown tergites; cheliceral groove with eleven denticles; seven spinnerets. Measurements: BL 15.40, CL 7.70, CW 6.80, OL 8.00, OW 5.55; ALE > PLE > PME > AME; leg I $23.05(6.05+3.10+4.90+6.10+2.90)$, leg II $24.46(6.48+3.08+5.05+6.80$ $+3.05)$, leg III $26.36(6.10+3.20+5.05+8.20+3.81)$, leg IV $32.70(8.00+3.50+$ $6.75+9.70+4.75)$.

Palp. Prolateral side of paracymbium unpigmented and unsclerotised, numerous setae and spines at the tip of paracymbium (Fig. 12A-C). Contrategulum with 


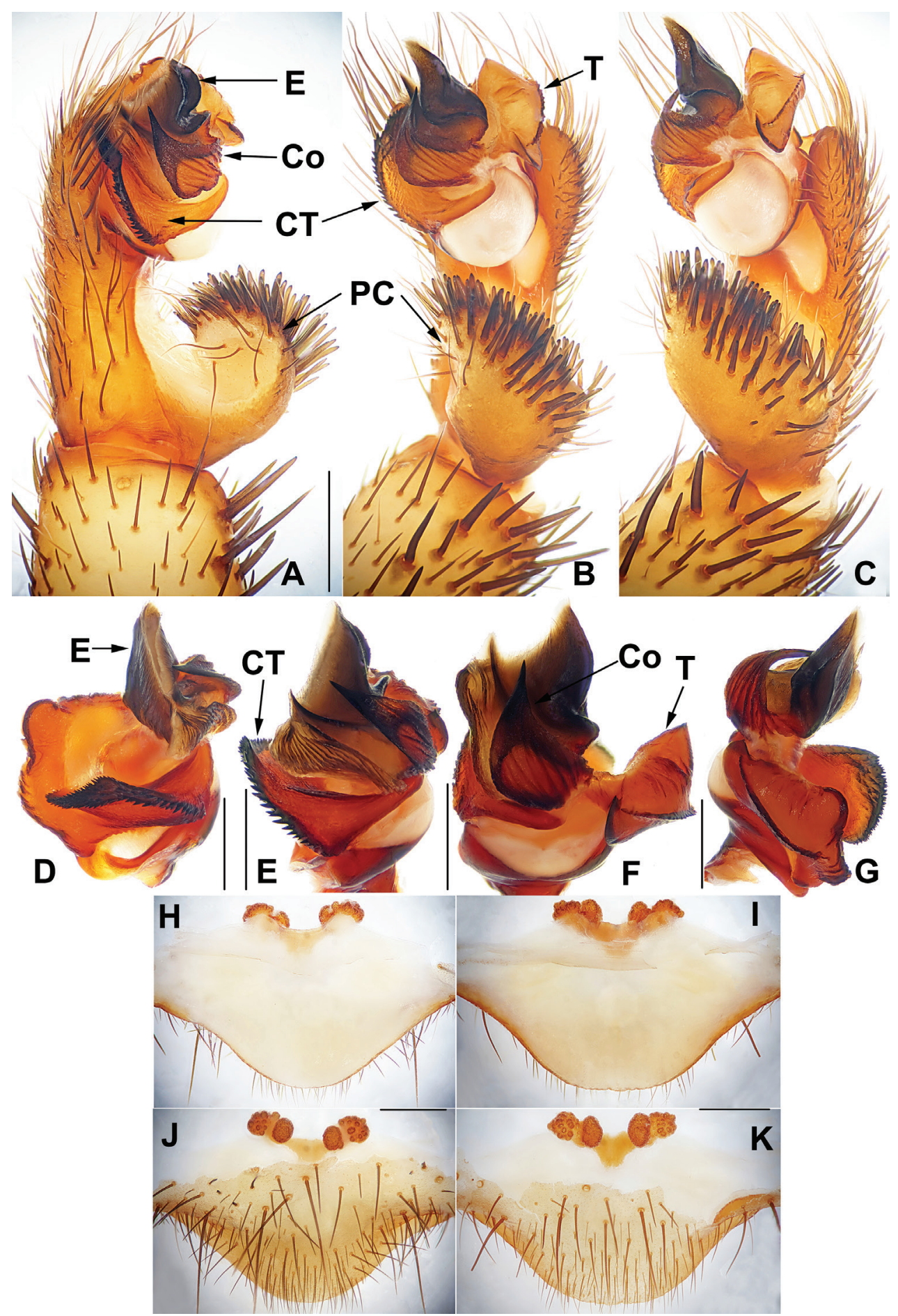

Figure I 2. Male and female genital anatomy of Heptathela sumiyo sp. nov. A-C 3293 (holotype, short for XUX-2013-293) D-G 3292 H, J 3288 I, K 3296 A palp prolateral view B palp ventral view C palp retrolateral view D-G palp distal view $\mathbf{H}, \mathbf{I}$ vulva dorsal view J, $\mathbf{K}$ vulva ventral view. Scale bar: $0.5 \mathrm{~mm}$. 
serrated margin (Fig. 12A, D, E). Tegulum wide with dentate dorsal extension of terminal apophysis (Fig. 12F, G) and blunt terminal apophysis (Fig. 12B, C, F, G). Conductor sclerotised and rugose, with several folds and a spiniform apex (Fig. $12 \mathrm{~A}-\mathrm{B}, \mathrm{E}-\mathrm{F})$. Embolus largely sclerotised, with a wide and flat opening, the distal part slightly sclerotised, and narrow and deep saddle-shaped in the prolateral view (Fig. 12A, D-F).

Females $(N=6)$. Carapace and opisthosoma colour as in male; cheliceral groove with 13 or 14 pronounced denticles; tergites similar to those of male; seven or eight spinnerets. Measurements: BL 11.70-15.60, CL 6.25-7.55, CW 5.40-6.43, OL 5.40-8.80, OW 4.00-7.35; ALE > PLE > PME > AME; palp $12.90(4.50+2.25+$ $2.70+3.45), \operatorname{leg}$ I $14.44(4.55+2.68+2.55+3.08+1.58)$, leg II $13.93(4.09+2.49$ $+2.35+3.20+1.80)$, leg III $15.61(4.40+2.70+2.68+3.80+2.03)$, leg IV 22.45 $(6.20+3.20+4.15+6.10+2.80)$.

Female genitalia. A pair of indistinct depressions on the ventro-lateral part of genital atrium (Fig. 12J, K). Two pairs of receptacular cluster along the anterior margin of bursa copulatrix, the medians ovate, the laterals tuberculate, similar or slightly larger than inners, without genital stalks (Fig. 12H-K).

Etymology. The species epithet, a noun in apposition, refers to the type locality.

Distribution. The species is known from the Japanese island Amamioshima (Fig. 1C).

\section{Heptathela uken sp. nov.}

http://zoobank.org/C538969C-60CB-4184-B388-E8958807AF33

Fig. 13

Type material. Holotype: JAPAN · Õ ; Kagoshima-ken, Amami, Uken-son, Oshimagun, Road No. 85, Redsoil Park; 28.24N, 129.34E; alt. 260 m; 15 September 2013; D. Li and B. Wu leg.; XUX-2013-297.

Paratypes: JAPAN · 2 ఏో, 2 우; same data as for holotype; XUX-2013-298,

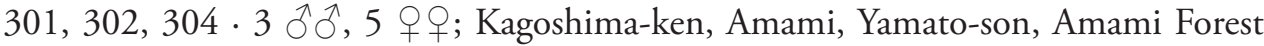
Park; 28.31N, 129.33E; alt. 300 m; 17 September 2013; D. Li and B. Wu leg.; XUX2013-305 to 314 .

Diagnosis. Males of $H$. uken sp. nov. can be distinguished from those of $H$. kane$n o i$ by the spiniform conductor apex (Fig. 13A, B, D, E), from those of $H$. amamiensis by the dorsal extension of tegular terminal apophysis without dentation (Fig. 13F, G). Females of H. uken sp. nov. cannot be diagnosed from those of the other Amami group Heptathela species morphologically (Fig. $13 \mathrm{H}-\mathrm{M}$ ), only by the following unique nucleotide substitutions in the standard DNA barcode alignment: T (161), T (191), G (227), C (236), T (287), T (297), A (299), C (389), T (395), G (413), C (416), G (503), C (509), C (510), T (527), T (558), G (560), G (569), C (578), T (584), C (596), T (614), G (629), G (635), C (650), C (665).

Description. Male (Holotype). Carapace brown; opisthosoma light brown, with dark brown tergites; cheliceral groove with eight denticles; seven spinnerets. Measure- 


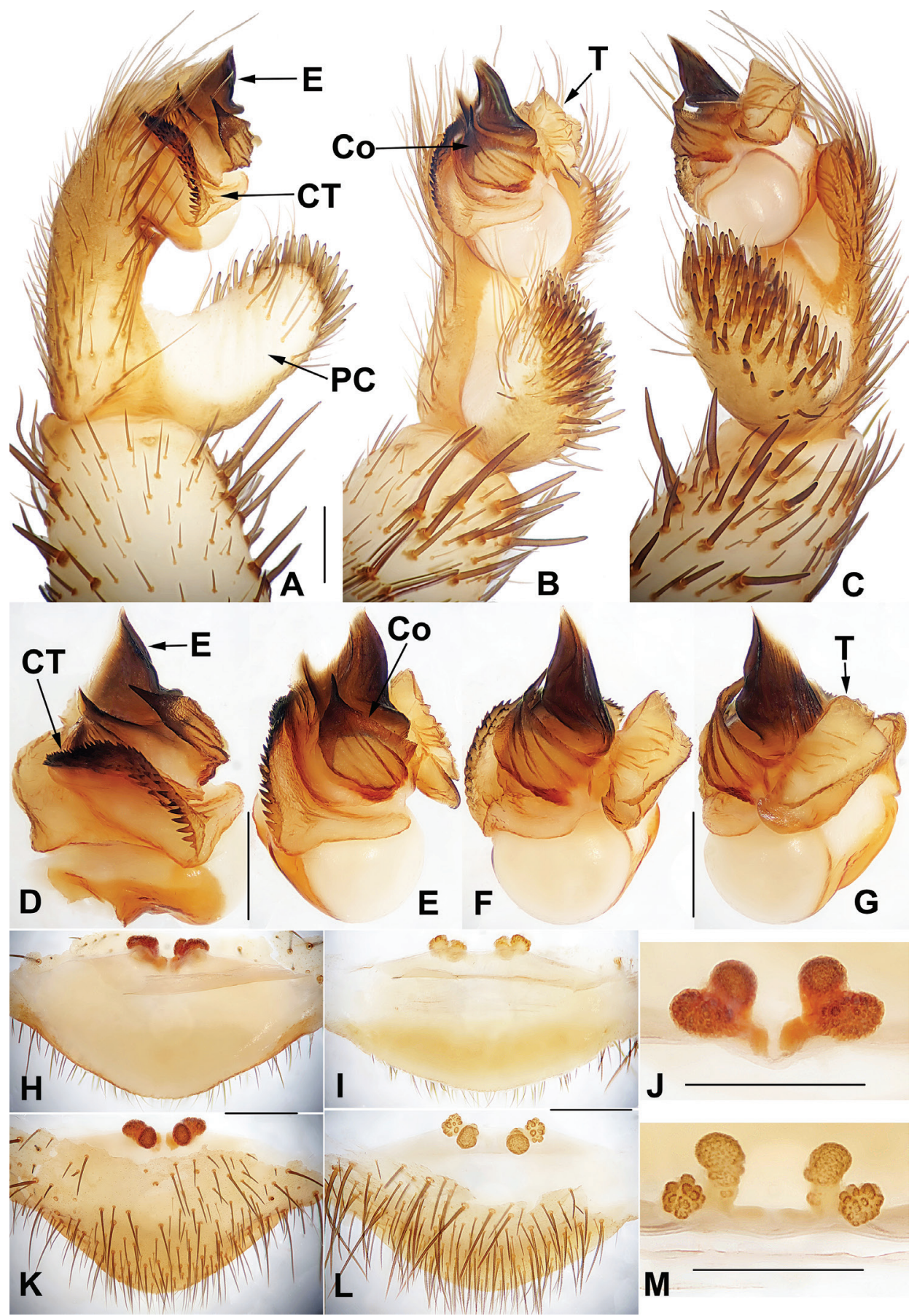

Figure 13. Male and female genital anatomy of Heptathela uken sp. nov. A-G 3297 (holotype, short for XUX-2013-297) H, K, J 3301 I, L, M 3309 A palp prolateral view B palp ventral view $\mathbf{C}$ palp retrolateral view D-G palp distal view $\mathbf{H}$, I vulva dorsal view K, $\mathbf{L}$ vulva ventral view J, M vulva distal view; 3297 , 3301: Uken-son, Amamioshima; 3309: Yamato-son, Amamioshima. Scale bar: 0.5 mm. 
ments: BL 11.60, CL 6.10, CW 5.60, OL 6.00, OW 4.30; ALE > PLE > PME > AME; leg I $17.47(4.80+2.28+3.67+4.42+2.30)$, leg II $17.95(4.65+2.30+3.60+4.90$ $+2.50)$, leg III $18.80(4.45+2.40+3.55+5.20+3.20)$, leg IV $23.60(5.60+2.45+$ $4.45+7.20+3.90)$.

Palp. Prolateral side of paracymbium unpigmented and unsclerotised, numerous setae and spines at the tip of paracymbium (Fig. 13A-C). Contrategulum with serrated margin (Fig. 13A, B, D, F). Tegulum with smooth dorsal extension of terminal apophysis (Fig. 13F, G). Conductor wide, sclerotised and rugose, with several folds and a spiniform apex (Fig. 13A, B, D, F). Embolus sclerotised, wide with a flat opening, and wide saddle-shaped in the prolateral view (Fig. 13A, B, D, E).

Females $(N=8)$. Carapace and opisthosoma colour as in male; cheliceral groove with 13-15 pronounced denticles; tergites similar to male; 7 or 8 spinnerets. Measurements: BL 11.80-16.00, CL 5.90-8.26, CW 5.20-7.20, OL 6.00-8.10, OW 4.606.00; ALE > PLE > PME > AME; palp $10.04(3.05+1.89+2.30+2.80)$, leg I 12.05 $(3.90+2.10+2.25+2.40+1.40)$, leg II $11.30(3.55+2.05+1.80+2.45+1.45)$, leg III $12.42(3.60+2.05+2.10+3.00+1.67)$, leg IV $18.33(5.15+2.40+3.30+$ $4.78+2.70)$.

Female genitalia. A pair of indistinct depressions on the ventro-lateral part of genital atrium (Fig. 13K). Two pairs of receptacular clusters along the anterior margin of bursa copulatrix, the inners almost globose, with short genital stalks in distal view, the laterals tuberculate, without genital stalks (Fig. 13H-M).

Etymology. The species epithet, a noun in apposition, refers to the type locality.

Distribution. The species is known from the Japanese island Amamioshima (Fig. 1C).

\section{The Okinawa group}

Diagnosis. The males of the Okinawa group differ from those of the Kyushu group by the semi-elliptic contrategulum (Fig. 14H-J), and from those of the Amami group by the contrategulum whose margin is only weakly serrated, is curved in the middle, and is proximally serrated and distally smooth (Fig. 14I, J). The females of the Okinawa group resemble those of the Kyushu group, but differ from those of the Amami group by distinctly paired depressions on the ventro-lateral part of the genital atrium (Fig. 14C, D).

Monophyly. The Bayesian analyses based on concatenated two genes and two partitions (for details, see Xu et al. 2019) suggested the Okinawa clades are paraphyly (Fig. $3)$. Therefore, in this study, Okinawa group includes all species from Okinawa except H. helios, which is sister to all the other Heptathela species (Fig. 3).

Composition. H. yanbaruensis Haupt, 1983, H. aha sp. nov., H. gayozan sp. nov., H. kubayama sp. nov., H. mae sp. nov., H. otoha sp. nov., H. shuri sp. nov., H. tokashiki sp. nov., H.unten sp. nov., and H. crypta sp. nov.

Distribution. Okinawajima, Iheyajima, Tokashikijima (Fig. 1C). 


\section{Heptathela yanbaruensis Haupt, 1983}

Fig. 14

Heptathela kimurai yanbaruensis Haupt, 1983: 284 (holotype: male, from Yona, Okinawa, Japan, collected by J. Haupt on 15 April 1977, deposited in ZMH, where the type may be lost (Dunlop et al. 2014); Haupt 1984: 166; Haupt 2003: 69. Heptathela yanbaruensis: Ono 2009: 80; Ono and Ogata 2018: 28, 480.

Diagnosis. Males of $H$. yanbaruensis can be distinguished from those of $H$. helios by the contrategulum that is distinctly curved in the middle (Fig. 14I, J), and from those of $H$. unten sp. nov. and H. crypta sp. nov. by the conductor that is longer than its width (Fig. 14I, J). Females of $H$. yanbaruensis can be distinguished from those of $H$. shuri sp. nov. by the wide and straight posterior margin of the genital atrium (Fig. 14A, C). Heptathela yanbaruensis can also be diagnosed from all other Heptathela species of the Okinawa group by the following unique nucleotide substitutions in the standard DNA barcode alignment: $\mathrm{G}$ (53), T (327), A (356), A (443).

Description. Males $(N=3)$. Carapace yellow brown; opisthosoma light brown, with dark-spotted tergites close to each other; cheliceral groove with 10-12 denticles; seven spinnerets. Measurements: BL 8.53-9.22, CL 4.21-4.57, CW 3.71-3.75, OL 4.20-4.48, OW 2.30-2.80; ALE > PLE > PME > AME; leg I $14.28(4.15+1.70+$ $3.05+3.56+1.82)$, leg II $15.15(3.97+1.70+3.07+4.13+2.28)$, leg III 15.24 $(4.00+1.68+3.08+4.10+2.38), \operatorname{leg}$ IV $19.78(4.75+1.37+3.91+6.45+3.30)$.

Palp. Prolateral side of paracymbium unpigmented and unsclerotised, numerous setae and spines at the tip of paracymbium (Fig. 14E-G). Contrategulum margin obviously curved in the middle, the contrategulum margin proximally serrated and distally smooth (Fig. 14H-J). Tegulum wide with a dentate dorsal extension of terminal apophysis (Fig. 14J, K), blunt terminal and marginal apophysis (Fig. 14J, K). Conductor oval, with weakly serrated margin, and a fold in prolateral view (Fig. 14H-J). Embolus sclerotised, with a wide opening, the distal margin slightly sclerotised, and with a wide saddle-shaped margin in the retrolateral view (Fig. 14I-K).

Females $(N=15)$. Carapace and opisthosoma colour as in male; cheliceral groove with 11-14 pronounced denticles; tergites similar to male; seven spinnerets. Measurements: BL 7.78-10.33, CL 3.90-5.10, CW 3.40-4.23, OL 3.90-6.00, OW 2.80-4.70; ALE $>$ PLE > PME > AME; palp $8.70(2.96+1.51+2.11+2.12)$, leg I $10.20(3.12+$ $1.75+1.93+2.12+1.28)$, leg II $9.97(3.05+1.72+1.77+2.13+1.30)$, leg III 10.05 $(2.91+1.78+1.48+2.47+1.41)$, leg IV $14.77(4.08+2.10+2.51+3.97+2.11)$.

Female genitalia. A pair of depressions on the ventro-lateral part of the genital atrium (Fig. 14C, D). The posterior margin of genital atrium wide and straight (Fig. 14A-D). Paired receptacular clusters along the anterior margin of bursa copulatrix, divided into two parts, the size of inner ones similar to that of laterals, with several granules, with short genital stalks (Fig. 14A-D).

Material examined. JAPAN · $3 \hat{\partial} \hat{\partial}, 9$ 우; Okinawa-ken, Kunigami-son, Yona, Tropical Biosphere Research Centre field station, University of the Ryukyu; 26.76N, 128.22E; alt. 20 m; 18 December 2012; D. Li, F.X. Liu and X. Xu leg.; XUX-2012-310 

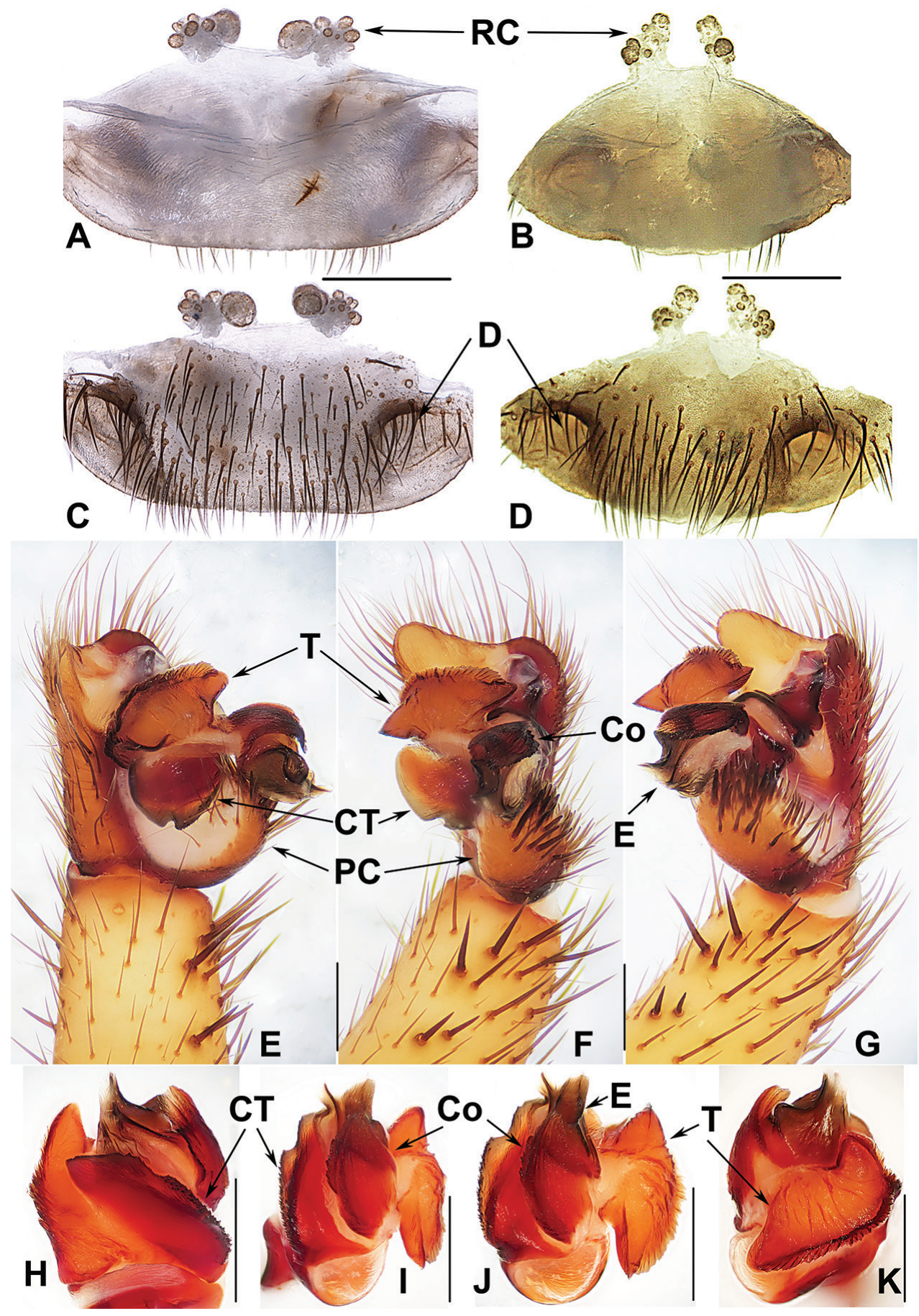

Figure 14. Male and female genital anatomy of Heptathela yanbaruensis Haupt, 1983 A, C 2315 (short for XUX-2012-315) B, D 2441 E-G 2322B H-K 2311 A, B vulva dorsal view C, D vulva ventral view E palp prolateral view $\mathbf{F}$ palp ventral view $\mathbf{G}$ palp retrolateral view $\mathbf{H}-\mathbf{K}$ palp distal view; 2311, 2315, 2322B: Yona, Okinawajima; 2441: Taiho Dam, Okinawajima. Scale bar: $0.5 \mathrm{~mm}$. 
to 322B - 6 우영 Okinawa-ken, Ogimi-son, Taiho Dam; 26.65N, 128.16E; alt. 80 m; 24 December 2012; D. Li, F.X. Liu and X. Xu leg.; XUX-2012-441 to 446.

Distribution. The species is endemic to the Japanese island Okinawajima (Fig. 1C).

\section{Heptathela aha sp. nov.}

http://zoobank.org/4F130218-5900-4688-AF57-F018C26E34C8

Fig. 15

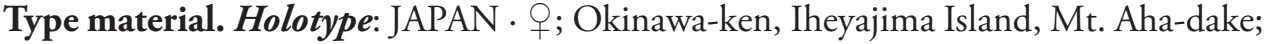
27.02N, 127.93E; alt. 10 m; 26 December 2012; D. Li, F.X. Liu and X. Xu leg.; XUX2012-502.

Paratypes: JAPAN - 6 우; same data as for holotype; XUX-2012-504 to 519.

Diagnosis. Females of $H$. aha sp. nov. cannot be distinguished morphologically from those of $H$. gayozan sp. nov. but can be distinguished from those of $H$. kubayama sp. nov. by the receptacular clusters without genital stalks; and from those of $H$. mae sp. nov. by the inner receptacular clusters similar to or larger than laterals (Fig. 15A-F). Heptathela aha sp. nov. can also be diagnosed from all other Okinawa group Heptathela species by the following unique nucleotide substitutions in the standard DNA barcode alignment: C (41), C (179), G (182), G (233), T (248), G (251), T (326), A (347), G (359), C (473), C (492), A (536), T (651).

Description. Female (Holotype). Carapace brown; opisthosoma brown, brown tergites with black plaques; cheliceral groove with 12 pronounced denticles; seven spinnerets. Measurements: BL 12.60, CL 5.55, CW 5.00, OL 6.82, OW 5.50; ALE > PLE > PME > AME; palp $9.90(3.40+1.71+2.18+2.61)$, leg I $11.34(3.31+2.05$ $+2.31+2.35+1.32)$, leg II $11.65(3.51+1.98+2.03+2.53+1.60)$, leg III 11.92 $(3.30+1.90+2.05+2.97+1.70), \operatorname{leg}$ IV $16.96(4.88+2.22+2.98+34.45+2.43)$.

Female genitalia. A pair of depressions on the ventro-lateral part of the genital atrium (Fig. 15D-F). A pair of receptacular clusters along the anterior margin of bursa copulatrix, divided into two parts, both without genital stalks (Fig. 15A-F).

Male. Unknown.

Etymology. The species epithet, a noun in apposition, refers to the type locality.

Distribution. The species is endemic to the Japanese island Iheyajima (Fig. 1C).

\section{Heptathela gayozan sp. nov.} http://zoobank.org/6DAC9A7B-50DD-4513-ACD6-B51AF264BBC2 Fig. 16

Type material. Holotype: JAPAN . + ; Okinawa-ken, Iheyajima Island, Mt. Gayozan; 27.02N, 127.97E; alt. 25 m; 27 December 2012; D. Li, F.X. Liu and X. Xu leg.; XUX2012-511.

Paratypes: JAPAN · 2 우; same data as for holotype; XUX-2012-513, 515. 


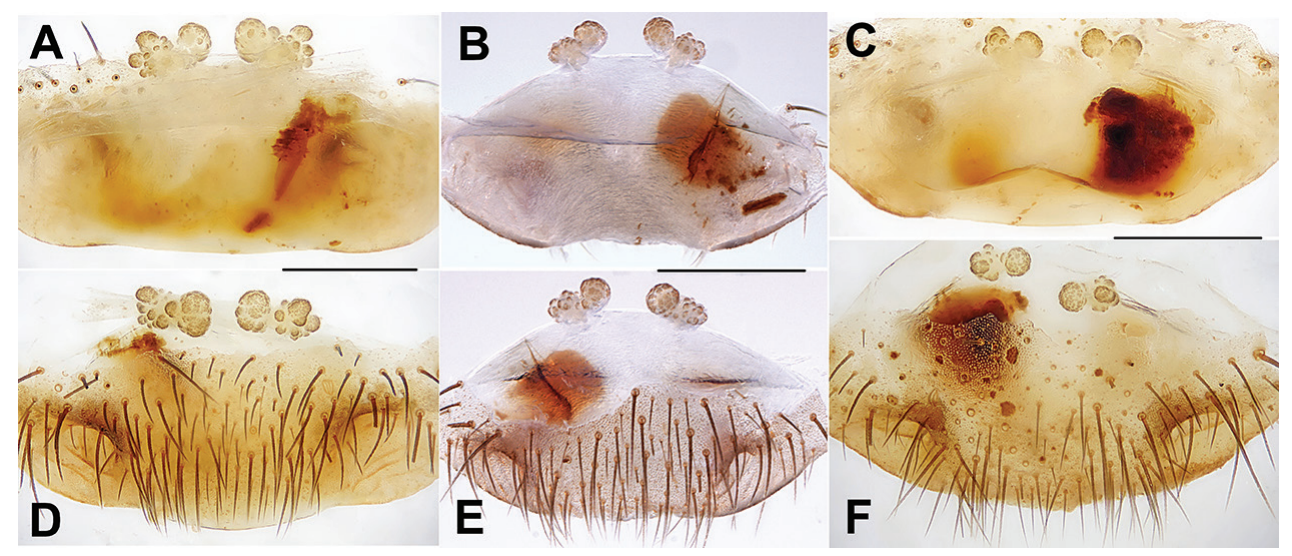

Figure 15. Female genital anatomy of Heptathela aha sp. nov. A, D 2502 (holotype, short for XUX2012-502) B, E 2505 C, F 2506 A-C vulva dorsal view D-F vulva ventral view. Scale bar: $0.5 \mathrm{~mm}$.

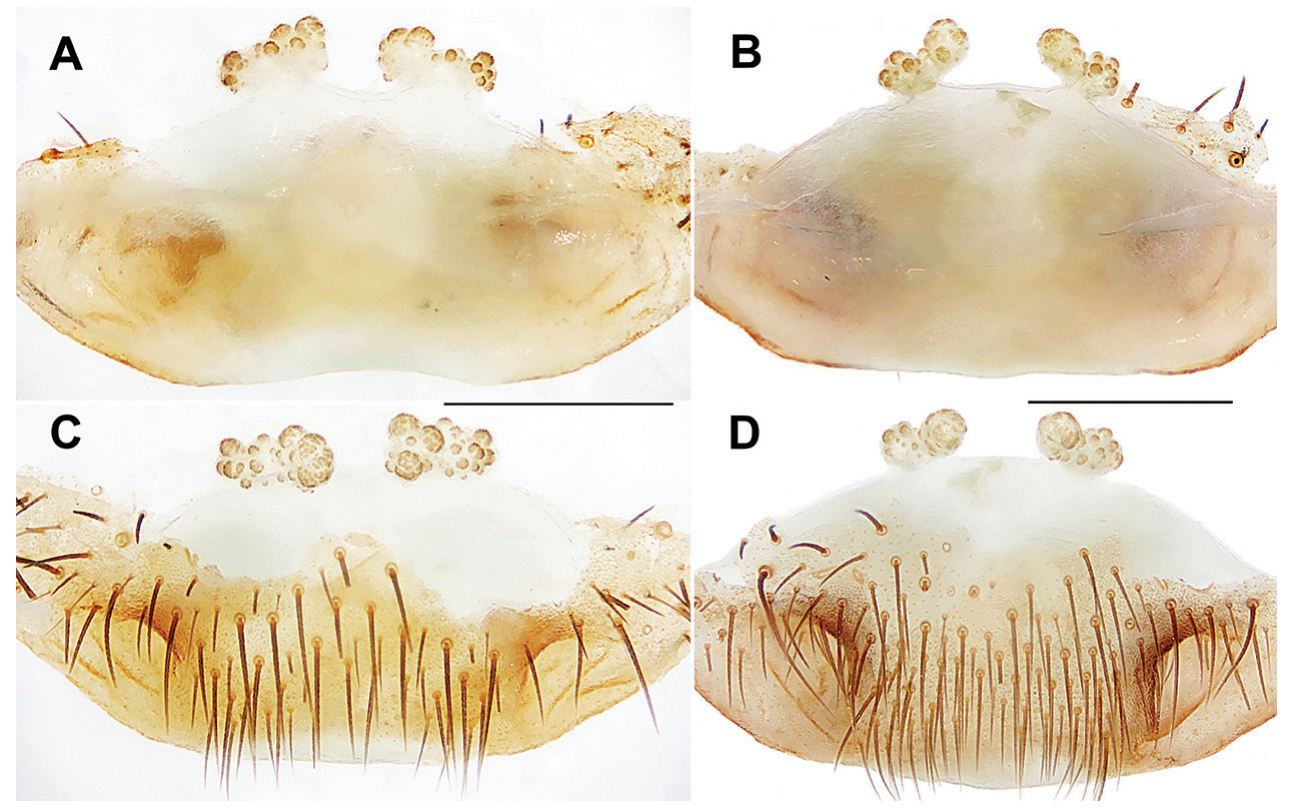

Figure 16. Female genital anatomy of Heptathela gayozan sp. nov. A, C 2511 (holotype, short for XUX2012-511) B, D 2513 A, B vulva dorsal view C, D vulva ventral view. Scale bar: $0.5 \mathrm{~mm}$.

Diagnosis. Females $H$. gayozan sp. nov. cannot be distinguished morphologically from those of $H$. aha sp. nov. but can be distinguished from those of $H$. kubayama sp. nov. by the receptacular clusters without genital stalks; and from those of $H$. mae sp. nov. by the inner receptacular clusters that are equal in size, or slightly larger than laterals (Fig. 16A-D). H. gayozan sp. nov. can be diagnosed from all other Okinawa group Heptathela species by the following unique nucleotide substitutions in the standard 
DNA barcode alignment: G (38), G (41), G (122), C (203), C (365), C (452), C (470), T (518), C (527), C (533), T (560), T (653).

Description. Female (holotype). Carapace brown; opisthosoma light brown, with dark brown tergites close to each other; cheliceral groove with 12 denticles; seven spinnerets. Measurements: BL 10.00, CL 4.40, CW 3.88, OL 4.84, OW 3.48; ALE > PLE > PME > AME; palp $7.32(2.72+1.50+1.80+1.30)$, leg I 9.61 $(2.95+1.62+1.82+1.97+1.25)$, leg II $9.38(2.80+1.58+1.71+2.07+1.22)$, leg III $9.60(2.65+1.55+1.62+2.28+1.50)$, leg IV $13.89(3.75+1.82+2.52$ $+3.75+2.05)$.

Female genitalia. A pair of depressions on the ventro-lateral part of genital atrium (Fig. 16C, D). A pair of receptacular clusters along the anterior margin of bursa copulatrix, divided into two parts, with several granules, without genital stalks (Fig. 16).

Male. Unknown.

Etymology. The species epithet, a noun in apposition, refers to the type locality.

Distribution. The species is endemic to the Japanese island Iheyajima (Fig. 1C).

\section{Heptathela kubayama sp. nov.}

http://zoobank.org/C8B09629-5191-447F-8C89-9953504F8E0D

Fig. 17

Type material. Holotype: JAPAN - + ; Okinawa-ken, Iheyajima Island, Mt. Kubayama Nature Conservation Area; 27.09N, 128.02E; alt. 85 m; 26 December 2012; D. Li, F.X. Liu and X. Xu leg.; XUX-2012-486.

Paratypes: JAPAN $\cdot 8$ + 9 ; same data as for holotype; XUX-2012-479, 481 to 485, 487 to 488.

Diagnosis. Females of $H$. kubayama sp. nov. can be distinguished from those of $H$. gayozan sp. nov. and $H$. mae sp. nov. by paired receptacular clusters with short genital stalks (Fig. 17A-B). Heptathela kubayama sp. nov. can also be diagnosed from all other Okinawa group Heptathela species by the following unique nucleotide substitutions in the standard DNA barcode alignment: G (239), G (329), G (353), C (359), G (443), G (602), G (647).

Description. Female (Holotype). Carapace brown; opisthosoma light brown, with dark brown tergites close to each other; cheliceral groove with 12 pronounced denticles; six spinnerets. Measurements: BL 10.20, CL 4.89, CW 4.18, OL 4.90, OW 3.60; ALE > PLE > PME > AME; palp $8.25(2.82+1.45+1.80+2.18)$, leg I $9.66(3.10+1.70+1.81+1.90+1.15)$, leg II $8.90(2.90+1.55+1.75+1.70+$ 1.00), leg III $9.59(2.81+1.65+1.60+2.13+1.40)$, leg IV $13.67(4.25+2.00+$ $2.00+3.54+1.88)$.

Female genitalia. A pair of depressions on the ventro-lateral part of the genital atrium (Fig. 17C, D). A pair of receptacular clusters along the anterior margin of bursa copulatrix, divided into two parts, with several granules; with short genital stalks (Fig. 17A-D). 


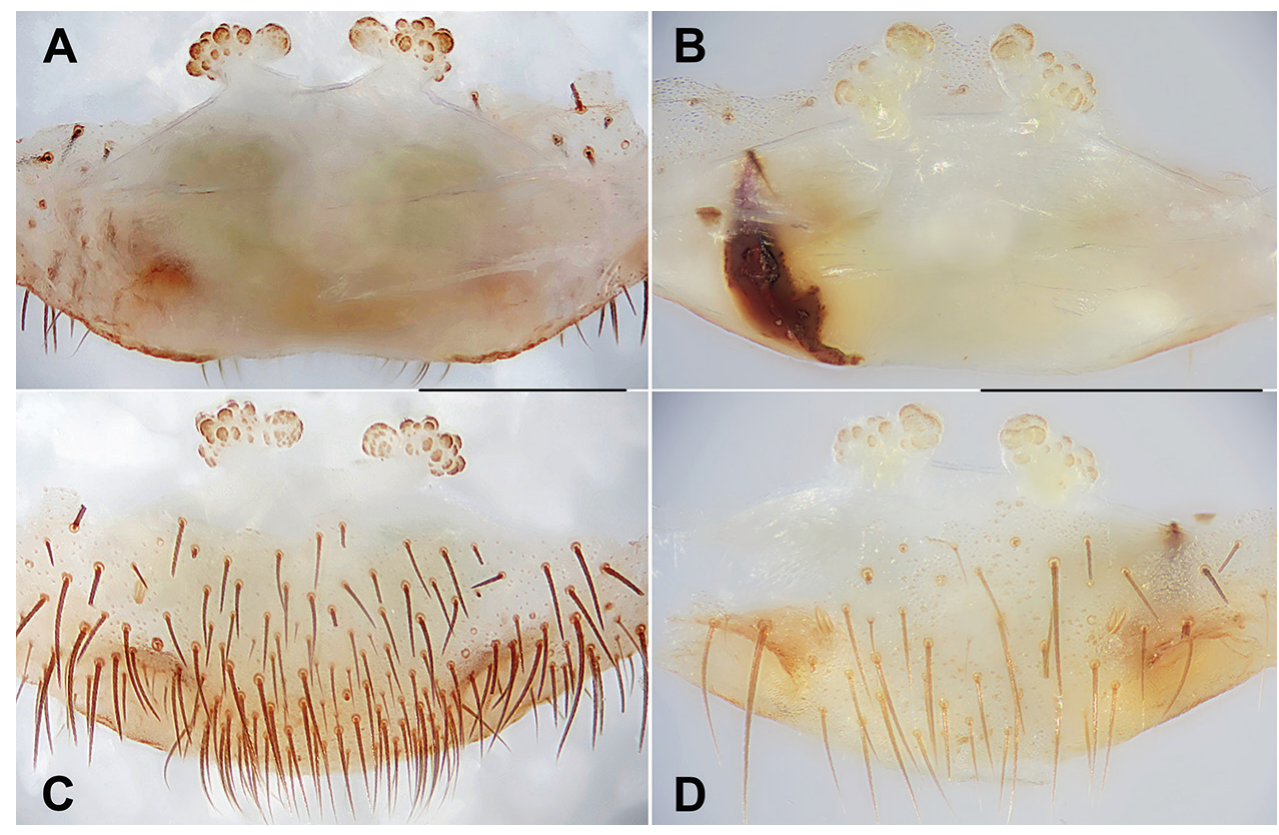

Figure 17. Female genital anatomy of Heptathela kubayama sp. nov. A, C 2486 (holotype, short for XUX-2012-486) B, D 2482 A, B vulva dorsal view C, D vulva ventral view. Scale bar: $0.5 \mathrm{~mm}$.

Male. Unknown.

Etymology. The species epithet, a noun in apposition, refers to the type locality. Distribution. The species is endemic to the Japanese island Iheyajima (Fig. 1C).

\section{Heptathela mae sp. nov.}

http://zoobank.org/45944FF0-D6B1-490B-ABCE-8CC5A933EDC3

Fig. 18

Type material. Holotype: JAPAN · + ; Okinawa-ken, Iheyajima Island, Mt. Mae-dake; 27.06N, 127.99E; alt. 10 m; 26 December 2012; D. Li, F.X. Liu and X. Xu leg.; XUX2012-497.

Paratypes: JAPAN . 5 우; same data as for holotype; XUX-2012-494 to 496, 498, 500 - 3우; Okinawa Prefecture, Iheyajima Island, Mt. Mae-dake; 27.06N, 127.99E; alt. 20 m; 10 May 2014; D. Li and B. Wu leg.; XUX-2014-079A to 079C.

Diagnosis. Females of $H$. mae sp. nov. can be distinguished from those of $H$. aha sp. nov. and $H$. gayozan sp. nov. by the lateral receptacular clusters being larger than the inner ones (Fig. 18B, D), and from those of H. kubayama sp. nov. by the receptacular clusters without short genital stalks (Fig. 18A-D). Heptathela mae sp. nov. can also be diagnosed from all other Okinawa group Heptathela species by the following unique nucleotide substitutions in the standard DNA barcode alignment: C (74), T (227), G (389), C (407), A (461), G (503), G (635). 


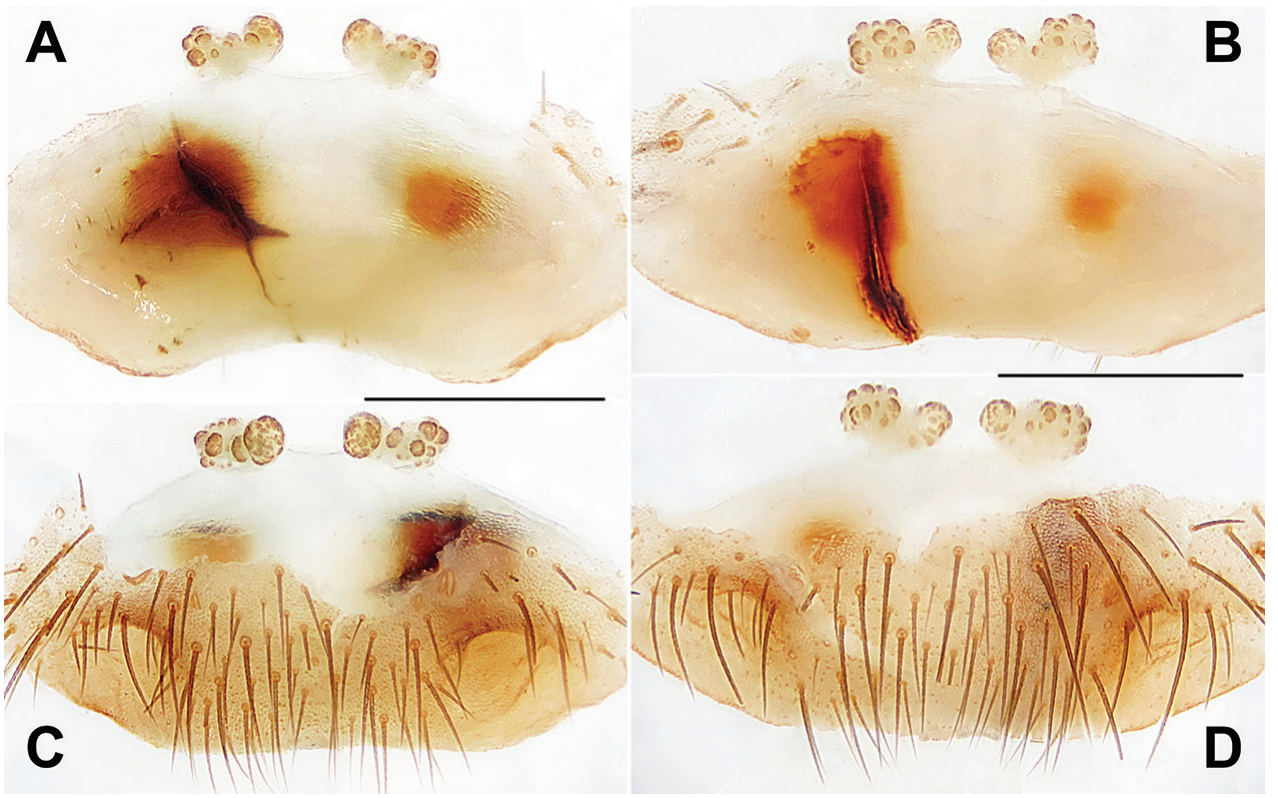

Figure 18. Female genital anatomy of Heptathela mae sp. nov. A, C 2497 (holotype, short for XUX2012-497) B, D 4079B A, B vulva dorsal view C, D vulva ventral view. Scale bar: $0.5 \mathrm{~mm}$.

Description. Female (Holotype). Carapace brown; opisthosoma brown, brown tergites with black plaques; cheliceral groove with 12 denticles; seven spinnerets. Measurements: BL 9.28, CL 4.48, CW 4.00, OL 5.12, OW 3.78; ALE > PLE > PME > AME; palp $7.80(2.70+1.32+1.71+2.07)$, leg I $9.25(3.00+1.52+1.80+1.78+$ 1.15), leg II $8.55(2.68+1.20+1.72+1.75+1.20)$, leg III $9.61(2.75+1.60+1.55$ $+2.31+1.40)$, leg IV $13.41(3.90+1.70+2.31+3.60+1.90)$.

Female genitalia. A pair of depressions on the ventro-lateral part of the genital atrium (Fig. 18C, D). A pair of receptacular clusters along the anterior margin of bursa copulatrix, divided into two parts, with several granules, without genital stalks, the posterior part of genital area incurved (Fig. 18A-D).

Male. Unknown.

Etymology. The species epithet, a noun in apposition, refers to the type locality.

Distribution. The species is endemic to the Japanese island Iheyajima (Fig. 1C).

\section{Heptathela otoha sp. nov.}

http://zoobank.org/2C41220D-A64D-4750-9C99-D5F12FB4543E

Fig. 19

Type material. Holotype: JAPAN - ; Okinawa-ken, Nakijin-son, Mt. Otoha-dake; 26.67N, 127.97E; alt. 80 m; 27 December 2012; D. Li, F.X. Liu and X. Xu leg.; XUX2012-535. 


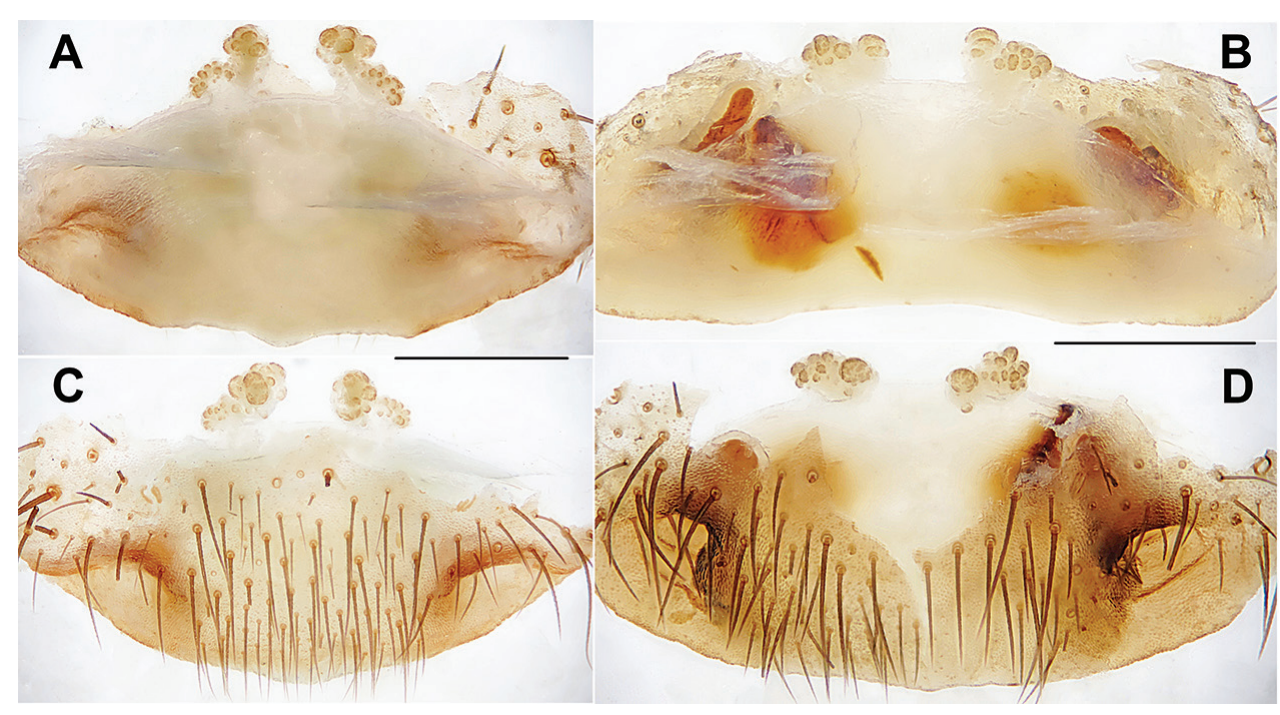

Figure 19. Female genital anatomy of Heptathela otoha sp. nov. A, C 2535 (holotype, short for XUX2012-535) B, D 4094 A, B vulva dorsal view C, D vulva ventral view. Scale bar: $0.5 \mathrm{~mm}$.

Paratypes: JAPAN · 3 우영 Okinawa Prefecture, Motobu-cho, Yamazato; 26.67N, 127.91E; alt. 160 m; 11 May 2014; D. Li and B. Wu leg.; XUX-2014-094, 097, 099 . 3 우; Okinawa Prefecture, Nakijin-son, Mt. Otoha-dake; 26.67N, 127.97E; alt. 100 m; 11 May 2014; D. Li and B. Wu leg.; XUX-2014-101, 102, 103.

Diagnosis. Females of $H$. otoha sp. nov. can be distinguished from those of $H$. yanbaruensis, $H$. unten sp. nov., and $H$. crypta sp. nov. by the inner receptacular clusters with several granules, albeit these inner clusters are difficult to be separated from the laterals (Fig. 19A-D). H. otoha sp. nov. can also be diagnosed from all other Okinawa group Heptathela species by the following unique nucleotide substitutions in the standard DNA barcode alignment: G (83), G (206), G (488).

Description. Female (Holotype). Carapace yellow brown; opisthosoma light brown, with brown and black-spotted tergites; cheliceral groove with 13 pronounced denticles; seven spinnerets. Measurements: BL 10.92, CL 4.75, CW 4.35, OL 6.60, OW 4.90; ALE > PLE > PME > AME; palp $8.30(2.78+1.39+1.90+2.23)$, leg I $9.80(3.05+1.65+1.95+1.95+1.20)$, leg II $9.21(2.90+1.50+1.81+1.90+1.10)$, leg III $9.84(2.70+1.70+1.62+2.35+1.47)$, leg IV $14.74(4.00+1.90+2.70+$ $4.03+2.11)$.

Female genitalia. A pair of depressions on the ventro-lateral part of genital atrium (Fig. 19C, D). Paired receptacular clusters along the anterior margin of bursa copulatrix, divided into indistinct two parts, but difficulty to separate, both with several granules, with very short genital stalks (Fig. 19A-D).

Male. Unknow.

Etymology. The species epithet, a noun in apposition, refers to the type locality.

Distribution. The species is endemic to the Japanese island Okinawajima (Fig. 1C). 


\section{Heptathela shuri sp. nov.}

http://zoobank.org/B74BEC88-F046-4583-80F3-E6BD2909BFAC

Fig. 20

Type material. Holotype: JAPAN - O; Okinawa-ken, Naha, Shuri, Sueyoshi Park; 26.23N, 127.72E; alt. 45 m; 17 December 2012; D. Li, F.X. Liu and X. Xu leg.; XUX-2012-309.

Paratype: JAPAN · 1 ; ; same data as for holotype; XUX-2012-308.

Diagnosis. Females of $H$. shuri sp. nov. can be distinguished from those of $H$. tokashiki sp. nov. by paired receptacular clusters with larger granules (Fig. 20AD). Heptathela shuri sp. nov. can also be diagnosed from all other Okinawa group Heptathela species by the following unique nucleotide substitutions in the standard DNA barcode alignment: G (89), G (131), C (134), C (396), C (510), C (512), A (524), T (641).

Description. Female (Holotype). Carapace yellow brown; opisthosoma brown, tergites with brown plaques; cheliceral groove with 12 pronounced denticles; seven spinnerets. Measurements: BL 10.78, CL 5.30, CW 4.12, OL 6.25, OW 4.40; ALE > PLE > PME > AME; palp $8.93(3.15+1.43+2.03+2.32)$, leg I $10.91(3.48+1.87$ $+2.05+2.21+1.30), \operatorname{leg}$ II $10.64(3.18+1.85+1.86+2.28+1.47), \operatorname{leg}$ III 11.26 $(3.03+1.81+1.77+2.90+1.75)$, leg IV $17.02(4.62+2.28+3.07+4.50+2.55)$.

Female genitalia. A pair of depressions on the ventro-lateral part of genital atrium (Fig. 20B, D). Paired receptacular clusters along the anterior margin of bursa copulatrix, divided into two parts, with several granules, the inner granules larger than laterals, both without genital stalks (Fig. 20A-D).

Male. Unknown.

Etymology. The species epithet, a noun in apposition, refers to the type locality.

Distribution. The species is endemic to the Japanese island Okinawajima (Fig. 1C).

\section{Heptathela tokashiki sp. nov.}

http://zoobank.org/A5A7B2B1-FE6F-4B42-AF06-48E7BDD937CB

Fig. 21

Type material. Holotype: JAPAN - $;$; Okinawa-ken, Tokashikijima Island, Aharen; 26.19N, 127.37E; alt. 100 m; 8 May 2014; D. Li and B. Wu leg.; XUX-2014-062.

Paratypes: JAPAN · 25 우; same data as for holotype; XUX-2014-046 to 063D . 3 우 ; same data as for holotype; 27 December 2012; D. Li, F.X. Liu and X. Xu leg.; XUX-2012-417, 421, 425.

Diagnosis. Females of $H$. tokashiki sp. nov. can be distinguished from $H$. shuri sp. nov. by the long inner receptacular clusters with several granules (Fig. 21A-D). Heptathela tokashiki sp. nov. can also be diagnosed from all other Okinawa group 


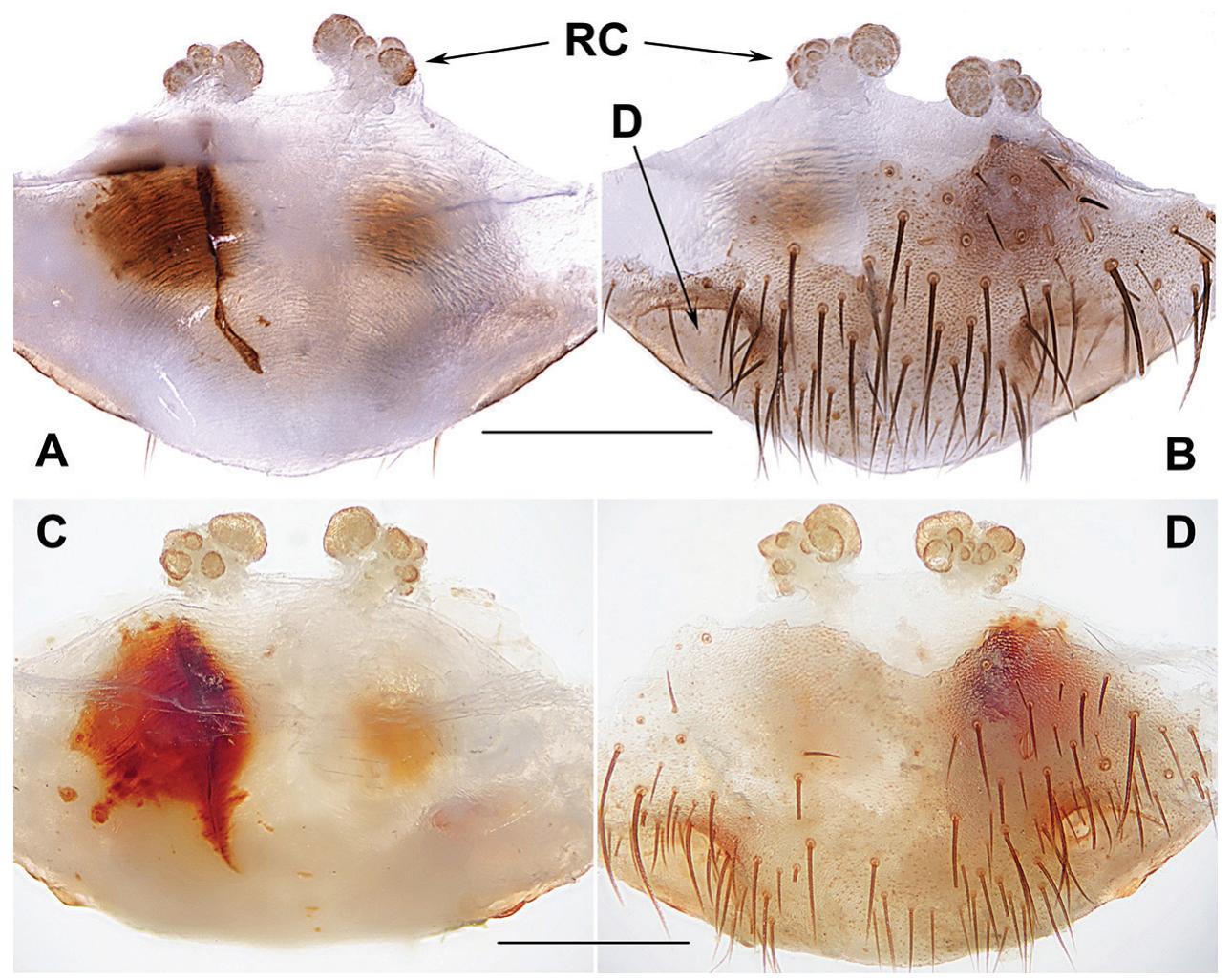

Figure 20. Female genital anatomy of Heptathela shuri sp. nov. A, B 2308 (short for XUX-2012-308) C, D 2309 (holotype) A, C vulva dorsal view B, D vulva ventral view. Scale bar: $0.5 \mathrm{~mm}$.

Heptathela species by the following unique nucleotide substitutions in the standard DNA barcode alignment: T (68), A (200), C (290), A (362).

Description. Female (Holotype). Carapace brown; opisthosoma dark brown, with dark brown tergites close to each other; cheliceral groove with eleven pronounced denticles; seven spinnerets. Measurements: BL 11.80, CL 5.15, CW 4.10, OL 6.80, OW 5.10; ALE > PLE > PME > AME; palp $8.60(3.05+1.50+1.95+2.10)$, leg I $10.50(3.35+1.85+2.15+2.10+1.05), \operatorname{leg}$ II $10.45(3.40+1.75+2.00+2.20+$ 1.10), leg III $10.95(3.20+1.85+1.80+2.60+1.50)$, leg IV $15.70(4.60+2.00+$ $2.80+3.90+2.40)$.

Female genitalia. A pair of depressions on the ventro-lateral part of the genital atrium (Fig. 21B, D). Paired receptacular clusters along the anterior margin of bursa copulatrix, divided into two parts, the inners longer than the laterals, both with several granules, without genital stalks (Fig. 21A, B).

Male. Unknown.

Etymology. The species epithet, a noun in apposition, refers to the type locality.

Distribution. The species is endemic to the Japanese island Tokashikijima (Fig. 1C). 


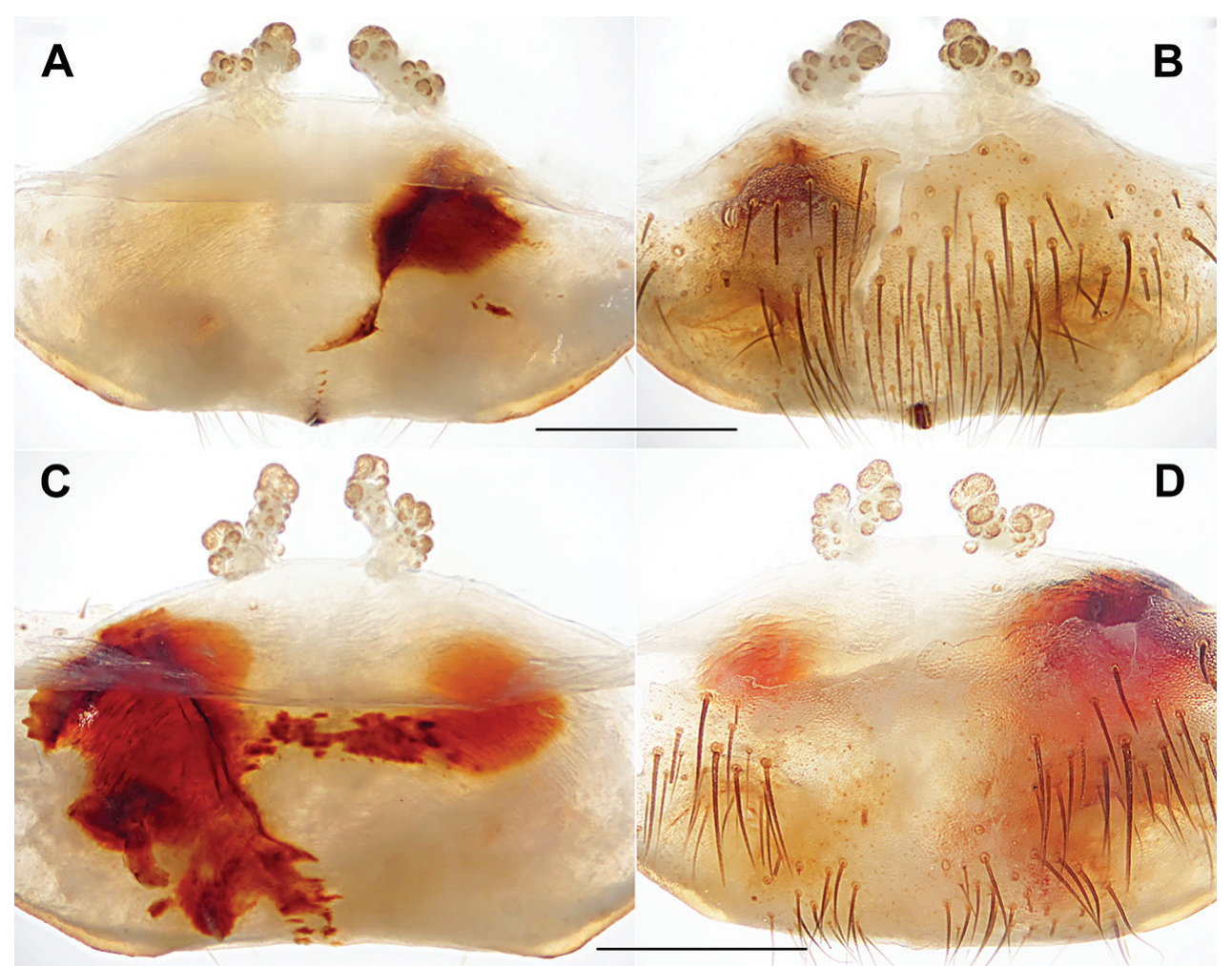

Figure 2 I. Female genital anatomy of Heptathela tokashiki sp. nov. A, B 4062 (holotype, short for XUX2014-062) C, D 4063 A, C vulva dorsal view B, D vulva ventral view. Scale bar: $0.5 \mathrm{~mm}$.

\section{Heptathela unten sp. nov.}

http://zoobank.org/65FF6B26-074B-4DC8-87F8-021268D23D87

Figs 22, 23

Type material. Holotype: JAPAN · $\widehat{~}$; Okinawa-ken, Nakijin-son, Unten Port; 26.68N, 128.00E; alt. 25 m; 27 December 2012; D. Li, F.X. Liu and X. Xu leg.; XUX-2012-522.

Paratypes: JAPAN $\cdot 1$ Oे, 2 우; same data as for holotype; XUX-2012-523, 527, 528A - 3 우; same data as for holotype; 10 May 2014; D. Li and B. Wu leg.; XUX2014-083, 083A, 083B.

Diagnosis. Males of $H$. unten sp. nov. can be distinguished from those of $H$. yanbaruensis by the blunt tegular marginal apophysis (Fig. 22A, C, D); from those of $H$. helios by the conductor with serrated margin and small tegular marginal apophysis (Fig. 22A, C). Females of $H$. unten sp. nov. cannot be distinguished morphologically form those of $H$. crypta sp. nov. (Fig. 23A-L). However, H. unten sp. nov. can be diagnosed from all other Okinawa group Heptathela species by the following unique nucleotide substitutions in the standard DNA barcode alignment: G (482), C (635). 


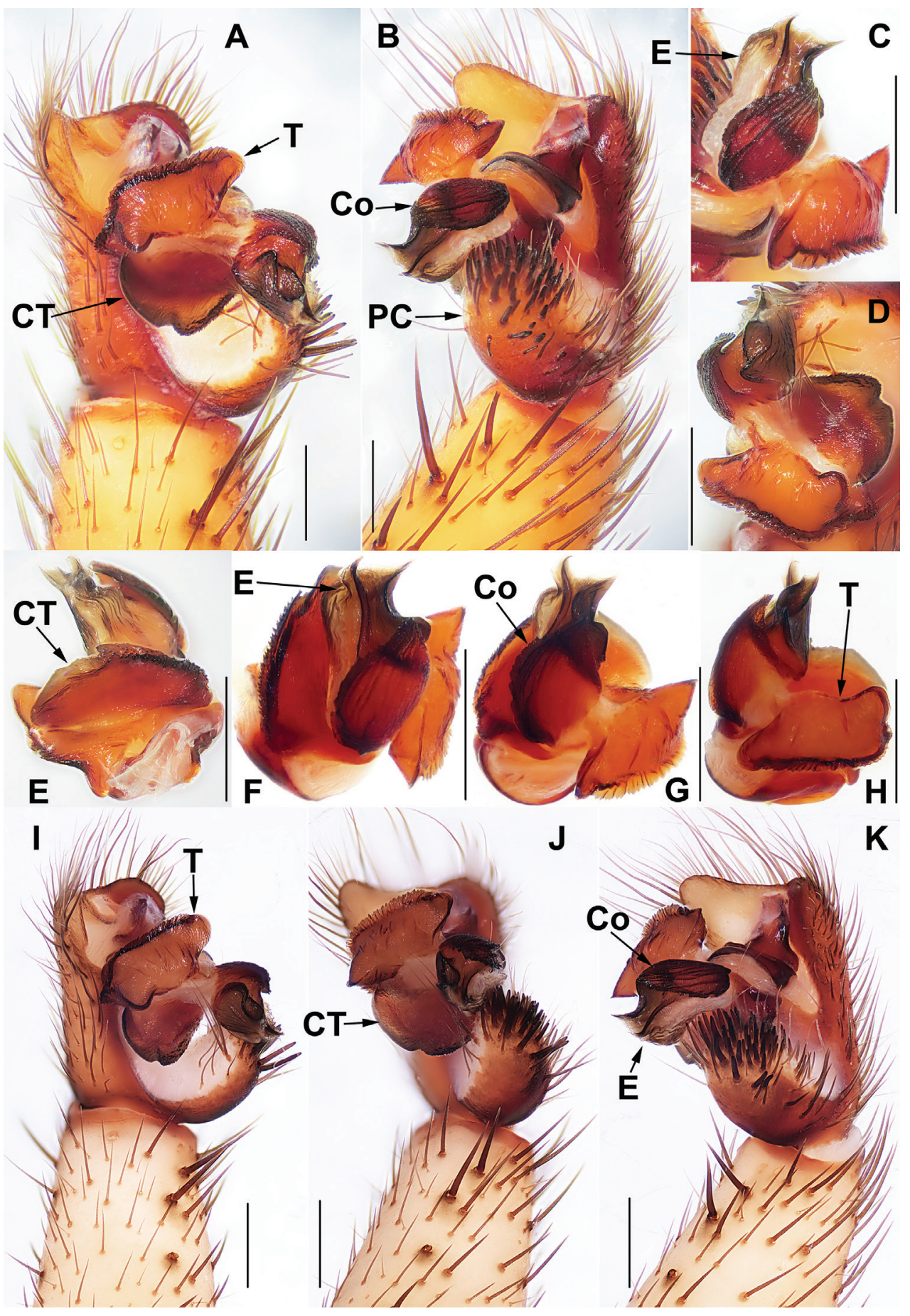

Figure 22. Male genital anatomy of Heptathela unten sp. nov. and H. crypta sp. nov. A-D Heptathela unten sp. nov., 2522 (holotype, short for XUX-2012-522) E-K H. crypta sp. nov. E-H 2328 (holotype) I-K 2460 A, I prolateral view B, K retrolateral view J ventral view C-H distal view; 2522: Unten Port, Okinawajima; 2328: Taira, Haneiji-Dam, Okinawajima; 2460: Yofuke, Okinawajima. Scale bar: $0.5 \mathrm{~mm}$. 


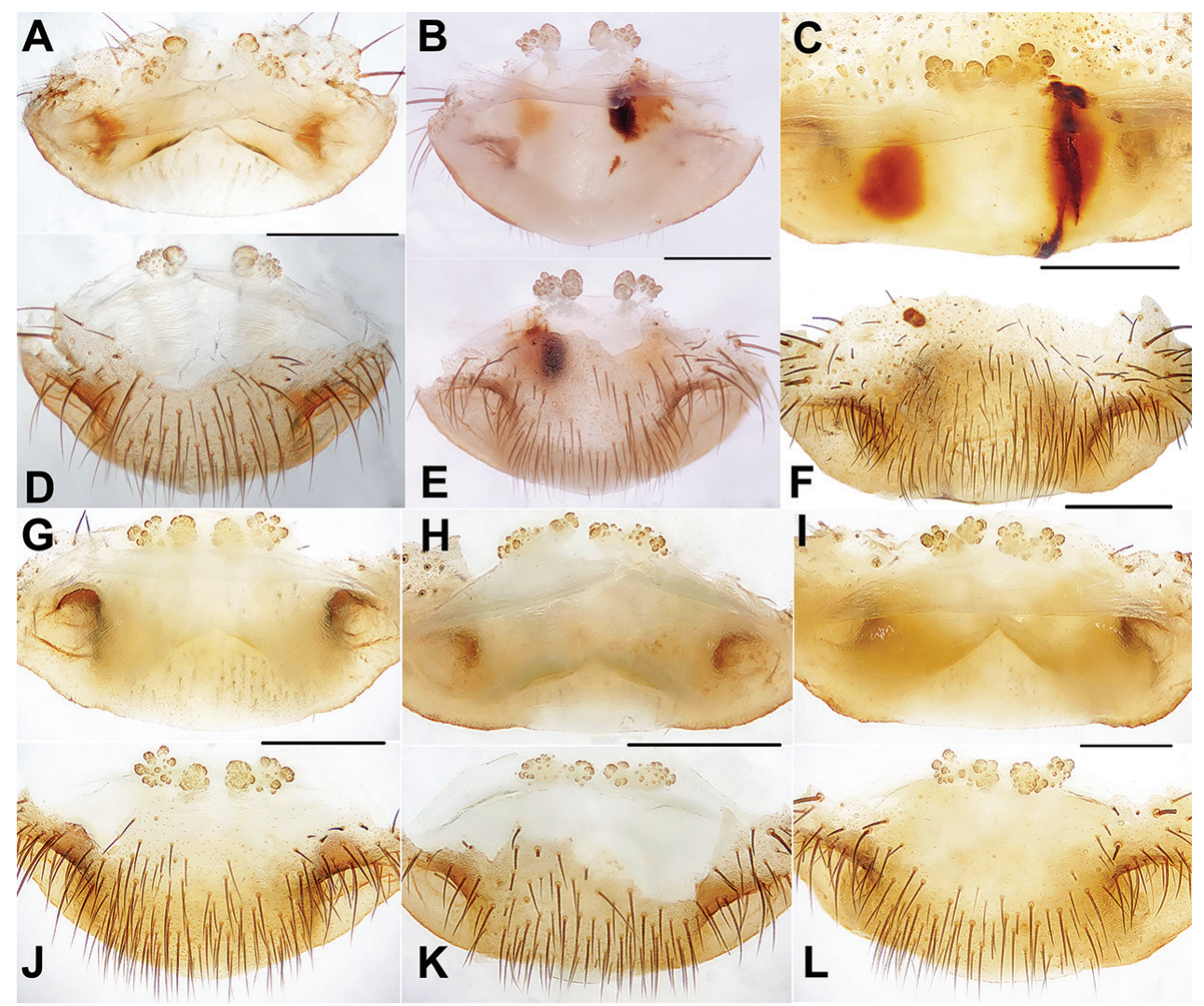

Figure 23. Female genital anatomy of Heptathela unten sp. nov. and H. crypta sp. nov. A, D 2527 B, E 4083A C, F 4027B G, J 2324 H, K 2327 I, L 2457 A-C, G-I dorsal view D-F, J-L ventral view; 2527, 4083A: Unten Port, Okinawajima; 4027B: Mt. Nago-dake, Okinawajima; 2324, 2327: Taira, Haneiji-Dam, Okinawajima; 2457: Yofuke, Okinawajima. Scale bar: $0.5 \mathrm{~mm}$.

Description. Male (Holotype). Carapace yellow brown; opisthosoma brown, with dark brown tergites close to each other; cheliceral groove with ten denticles; seven spinnerets. Measurements: BL 10.00, CL 5.05, CW 4.60, OL 4.60, OW 2.90; ALE > PLE $>$ PME > AME; leg I $15.71(4.48+1.93+3.30+4.00+2.00)$, leg II $16.59(4.45+$ $1.90+3.37+4.62+2.25)$, leg III $18.29(4.50+1.93+3.58+5.53+2.75)$, leg IV $23.40(5.82+2.28+4.52+7.30+3.48)$.

Palp. Prolateral side of paracymbium unpigmented and unsclerotised, numerous setae and spines at the tip of paracymbium (Fig. 22A, B). Contrategulum margin incurved nearly in the middle and the contrategulum divided into proximally serrated and distally smooth margins (Fig. 22A, D). Tegulum with wide dorsal extension of terminal apophysis (Fig. 22C, D), blunt terminal and small marginal apophysis (Fig. $22 \mathrm{~A}, \mathrm{C}, \mathrm{D})$. Conductor sclerotised and ovate, prolateral conductor with one or two shallow folds, and with a serrated margin (Fig. 22B, C). Embolus sclerotised, with a wide opening, the distal margin slightly sclerotised, and with a saddle-shaped margin in the retrolateral view (Fig. 22B, C). 
Females $(N=5)$. Carapace and opisthosoma colour as in male, dark brown tergites separated from each other; cheliceral groove with 12 or 13 pronounced denticles; seven or eight spinnerets. Measurements: BL 7.81-12.00, CL 3.60-4.55, CW 3.30-4.40, OL 4.10-7.60, OW 3.00-6.20; ALE > PLE > PME > AME; palp $6.64(2.19+1.22+$ $1.45+1.78)$, leg I $7.48(2.47+1.35+1.22+1.52+0.92)$, leg II $7.40(2.21+1.30+$ $1.21+1.65+1.03), \operatorname{leg}$ III $7.79(2.11+1.38+1.20+1.90+1.20), \operatorname{leg}$ IV $11.55(3.20$ $+1.55+2.10+3.05+1.65)$.

Female genitalia. A pair of depressions on the ventro-lateral part of genital atrium indistinct (Fig. 23D, E). Paired receptacular clusters along the anterior margin of bursa copulatrix, divided into two parts, the inners similar or smaller than the laterals, paired receptacular clusters tuberculate, inners with or without genital stalks (Fig. 23A, B, D, E).

Etymology. The species epithet, a noun in apposition, refers to the type locality, Unten Port.

Distribution. The species is endemic to the Japanese island Okinawajima (Fig. 1C).

\section{Heptathela crypta sp. nov.}

http://zoobank.org/62DDDCB8-8B43-4EFC-B42C-9BAA1E114A23

Figs 22, 23

Type material. Holotype: JAPAN · Ô; Okinawa-ken, Nago-shi, Haneiji-Dam, Taira; 26.59N, 128.03E; alt. 100 m; 18 December 2012; D. Li, F.X. Liu and X. Xu leg.; XUX-2012-328.

Paratypes: JAPAN · 1 त, 3 우; same data as for holotype; XUX-2012-324, 326,

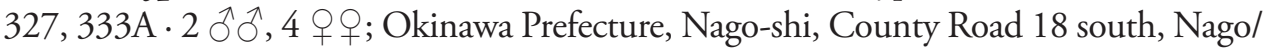
Yofuke; 26.57N, 128.01E; alt. 150 m; 24 December 2012; D. Li, F.X. Liu and X. Xu leg.; XUX-2012-457 to 462 - 3 우; Okinawa Prefecture, Nago-shi, Mt. Nago-dake; 26.58N, 128.01E; alt. 220 m; 06 May 2014; D. Li and B. Wu leg.; XUX-2014-027 to 027B.

Diagnosis. Males and females of $H$. crypta sp. nov. cannot be distinguished morphologically from $H$. unten sp. nov. (Figs 22A-K, 23A-L), but can be diagnosed from $H$. unten sp. nov. by the following unique nucleotide substitutions in the standard DNA barcode alignment: C (26), A (32), C (50), T (60), T (110), G (153), T (194), C (197), T (269), C (281), T (284), C (338), A (341), T (357), C (416), T (428), C (458), A (482), T (488), G (551), T (581), T (635), G (638), G (641), C (644), C (656), as well as from all other Okinawa group Heptathela species by the following unique nucleotide substitutions in the standard DNA barcode alignment: C (26), T (110), G (551), C (656).

Description. Male (Holotype). Carapace and opisthosoma description see $H$. unten sp. nov.; cheliceral groove with nine denticles of variable size; seven spinnerets. Measurements: BL 7.88, CL 4.01, CW 3.51, OL 4.23, OW 3.18; ALE > PLE > PME $>$ AME; leg I $10.15(3.60+1.50+2.38+1.00+1.67), \operatorname{leg}$ II $13.08(3.48+1.58+2.49$ $+3.53+2.00)$, leg III $14.27(3.38+1.55+2.65+4.30+2.39)$, leg IV $18.19(4.45+$ $1.63+3.50+5.50+3.11)$. 
Palp. Prolateral side of paracymbium unpigmented and unsclerotised, numerous setae and spines at the tip of paracymbium (Fig. 22I-K). Contrategulum margin incurved nearly in the middle, and the contrategulum divided into proximally serrated and distally smooth (Fig. 22E, F, I, J). Tegulum with wide dorsal extension of terminal apophysis (Fig. 22G, H, J), blunt terminal and small marginal apophysis (Fig. 22H, I). Conductor sclerotised and ovate, prolateral conductor with one or two shallow folds, and with a serrated margin (Fig. 22F, G, K). Embolus sclerotised, with a wide opening, the distal margin slightly sclerotised, and with a saddle-shaped margin in the retrolateral view (Fig. 22F, G, K).

Females $(N=10)$. Carapace and opisthosoma description see $H$. unten sp. nov.; chelicerae with promargin of cheliceral groove with 13-14 pronounced denticles of variable size; seven spinnerets. Measurements: BL 8.35-16.50, CL 4.07-5.10, CW 3.30-4.80, OL 4.70-6.80, OW 3.00-5.20; ALE > PLE > PME > AME; palp 7.70 $(2.87+1.13+1.68+2.02), \operatorname{leg}$ I $9.57(3.07+1.70+1.70+1.98+1.12)$, leg II 9.64 $(2.95+1.68+1.61+2.08+1.32)$, leg III $9.60(2.68+1.69+1.50+2.30+1.43)$, leg IV $14.21(4.00+1.92+2.55+3.83+1.91)$.

Female genitalia. A pair of depressions on the ventro-lateral part of genital atrium indistinct (Fig. 23F, J-L). Paired receptacular clusters along the anterior margin of bursa copulatrix, divided into two parts, the inners similar or smaller than the laterals, paired receptacular clusters tuberculate, without genital stalks (Fig. 23C, G-L).

Etymology. The species epithet, a noun in apposition, refers to the cryptic nature of this species discovery.

Distribution. The species is endemic to the Japanese island Okinawajima (Fig. 1C).

\section{Species not assigned to a group}

\section{Heptathela helios Tanikawa \& Miyashita, 2014}

Fig. 24

Heptathela helios Tanikawa \& Miyashita, 2014: 68 (holotype: male (NSMT-Ar 12851), from Kunigami-son, Okinawajima, Japan, collected by A. Tanikawa on 26 May 2010, matured on 9 September 2012, deposited in NMNS, examined).

Diagnosis. Males of $H$. helios can be distinguished from those of all other Okinawa group Heptathela species by the serrated contrategulum margin and the hooked tegular marginal apophysis, the ovate, indistinctly rugose conductor with a poorly serrated margin (Fig. 24D-G). Females of $H$. helios can be distinguished from those of all other Heptathela species by the receptacular clusters with the inner ones being smaller than the laterals, and the laterals with numerous small granulate tubercula (Fig. $24 \mathrm{H}-\mathrm{M}$ ). $H$. helios can also be diagnosed from all other Okinawa group Heptathela species by the following unique nucleotide substitutions in the standard DNA barcode alignment: T (11), T (35), G (47), A (56), C (59), A (95), T (104), A (131), T (140), T (179), 


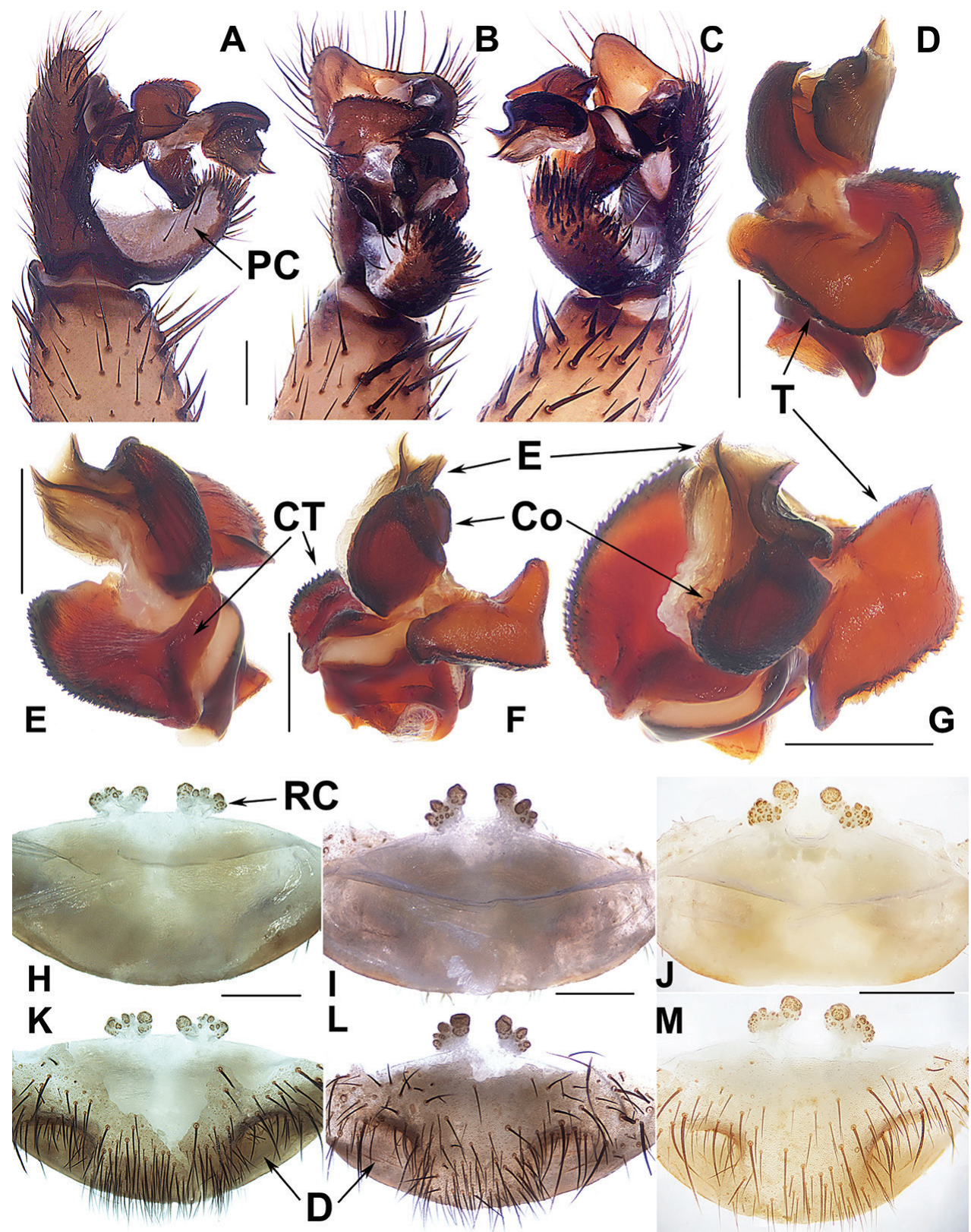

Figure 24. Male and female genital anatomy of Heptathela helio Tanikawa \& Miyashita, 2014 A-G 2440A (short for XUX-2012-440A) H, K 2432 I, L 2434 J, M 2437 A palp prolateral view B palp ventral view C palp retrolateral view D-G palp distal view $\mathbf{H}-\mathbf{J}$ vulva dorsal view $\mathbf{K}-\mathbf{M}$ vulva ventral view. Scale bar: $0.5 \mathrm{~mm}$.

C (188), C (215), G (221), C (242), C (266), C (273), C (299), C (300), C (304), T (359), G (380), T (413), A (422), T (425), T (431), G (479), G (480), G (491), G (506), C (543), T (546), G (548), C (551), C (596), T (662). 
Description. Male. Carapace and opisthosoma brown, with dark brown tergites; cheliceral groove with 14 denticles; tergites closed to each other; seven spinnerets. Measurements: BL 9.88, CL 5.30, CW 4.81, OL 4.58, OW 2.92; ALE > PLE > PME $>$ AME; leg I $16.56(4.55+1.90+3.48+4.33+2.30), \operatorname{leg}$ II $17.19(4.50+2.00+3.34$ $+4.68+2.67)$, leg III $18.35(4.35+1.97+3.40+5.53+3.10)$, leg IV $23.37(5.52+$ $2.10+4.47+7.48+3.80)$.

Palp. The bulb of the two male specimens relatively distorted. Prolateral side of paracymbium unpigmented and unsclerotised, numerous setae and spines at the tip of paracymbium (Fig. 24A-C). Contrategulum with a serrated margin (Fig. 24E, G). Tegulum with a dentate dorsal extension of terminal apophysis (Fig. 24F, G), blunt terminal and hook-like marginal apophysis (Fig. 24D, F, G). Conductor sclerotised, ovate, and wide, with indistinct rugae (Fig. 24E-G). Embolus sclerotised, with a wide opening, the distal margin slightly sclerotised, and with a saddle-shaped margin in the retrolateral view (Fig. 24D-G).

Females $(N=7)$. Carapace and opisthosoma colour as in male; cheliceral groove with 12-14 pronounced denticles; opisthosoma with 12 well-separated tergites; seven spinnerets. Measurements: BL 11.70-14.45, CL 5.29-6.70, CW 4.29-5.81, OL 6.54-8.18, OW 4.70-6.63; ALE > PLE > PME > AME; palp $12.29(4.20+2.03+$ $2.63+3.43), \operatorname{leg}$ I $14.31(4.51+2.40+2.65+3.08+1.67), \operatorname{leg}$ II $14.04(4.25+2.35$ $+2.53+3.11+1.80)$, leg III $13.75(3.92+2.40+2.48+2.67+2.28)$, leg IV 21.77 $(5.90+2.88+3.75+6.11+3.13)$.

Female genitalia. A pair of depressions on the ventro-lateral part of genital atrium (Fig. 24K-M). Paired receptacular clusters along the anterior margin of bursa copulatrix, divided into two parts, inner receptacular clusters smaller than laterals, laterals with several small tubercula, with short genital stalks (Fig. 24H-M).

Remarks. We identified the specimens collected from Ginama Dam, Okinawa, as $H$. helios based on evidence from morphology and COI barcode genetic distance compared with the male holotype and paratype (NSMT-Ar 12851, NSMT-Ar 12855) of $H$. helios in Tanikawa and Miyashita (2014). K2P and $p$-distances between Ginama Dam specimens and the holotype (NSMT-Ar 12851) were 3.2-3.4\% and 3.1-3.3\%, respectively, and those between Ginama Dam specimens and the paratype (NSMT-Ar $12855)$ were $1.8-2.1 \%$ and $1.7-2.1 \%$, respectively.

Material examined. JAPAN $\cdot 2 \hat{\delta} \widehat{\partial}, 8$ 우; Okinawa-ken, Kunigami-son, Ginama Dam; 26.84N, 128.26E; alt. 150 m; 24 December 2012; D. Li, F.X. Liu and X. Xu leg.; XUX-2012-432 to 440C.

Distribution. The species is endemic to the Japanese island Okinawajima (Fig. 1C).

\section{Acknowledgements}

We thank Zoltán Korsós, Mamoru Toda, and Bo Wu for assistance in the field, and the staff of the Centre for Behavioural Ecology and Evolution (CBEE, Hubei University) for all their help and support throughout this study. We acknowledge constructive and 
insightful comments on the manuscript from Ingi Agnarsson, Jason Bond, Rebecca Godwin, and Xiang Xu. We thank Gonzalo Giribet, Laura Leibensperger, Stefan Friedrich, Danilo Harms, and Jason A. Dunlop for information on the types and for facilitating loans. This study was supported in part by the grants from National Natural Sciences Foundation of China (NSFC-31601850; NSFC-31272324), the Hunan Provincial Natural Science Foundation of China (2017JJ3202), Singapore Ministry of Education AcRF Tier 1 grant (R-154-000-A52-114) to DL, the Japan Society of Promotion of Science (JSPS-21540487), the Slovenian Research Agency (P1-10236 and J1-6729), and the bilateral inter-governmental S\&T exchange project between China and Slovenia (12-8).

\section{References}

Bristowe WS (1933) The liphistiid spiders. With an appendix on their internal anatomy by J. Millot. Proceedings of Zoological Society of London 1932: 1015-1057. https://doi. org/10.1111/j.1096-3642.1932.tb01575.x

Bao YH, Yin CM, Xu X (2003) A new species of the genus Heptathela from China (Araneae, Liphistiidae). Acta Zootaxonomica Sinica 28: 459-460.

Bishop SC, Crosby CR (1932) A new species of the spider family Liphistiidae from China. Peking Natural History Bulletin 6(3): 5-7.

Brower AVZ (2010) Alleviating the taxonomic impediment of DNA barcoding and setting a bad precedent: names for ten species of 'Astraptes fulgerator' (Lepidoptera: Hesperiidae: Eudaminae) with DNA-based diagnoses. Systematics and Biodiversity 8: 485-491. https:// doi.org/10.1080/14772000.2010.534512

Chen XE, Gao JC, Zhu CD, Luo ZM (1988) A new species of the genus Heptathela from China (Araneae: Heptathelidae). Sichuan Journal of Agricultural Science 1988: 78-81.

Chen ZF, Zhang ZH, Zhu CD (1981) A new species of genus Heptathela. Journal of Hangzhou University 8: 305-308.

Chikuni Y (1989) Pictorial Encyclopedia of Spiders in Japan. Kaisei-sha Publishing Co., Tokyo, $310 \mathrm{pp}$.

Cook LG, Edwards RD, Crisp MD, Hardy NB (2010) Need morphology always be required for new species descriptions? Invertebrate Systematics 24: 322-326. https://doi. org/10.1071/IS10011

Dunlop JA, Steffensen C, Ono H (2014) Mesothele spiders in the Museum für Naturkunde Berlin. Arachnologische Mitteilungen 47: 35-40. https://doi.org/10.5431/aramit4705

Gertsch WJ (1967) A new liphistiid spider from China (Araneae: Liphistiidae). Journal of The New York Entomological Society 75: 114-118.

Gertsch WJ, Platnick NI (1979) A revision of the spider family Mecicobothriidae (Araneae, Mygalomorphae). American Museum Novitates 2687: 1-32.

Haupt J (1979) Lebensweise und Sexualverhalten der mesothelen Spinne Heptathela nishihirai n. sp. (Araneae, Liphistiidae). Zoologischer Anzeiger 202: 348-374.

Haupt J (1983) Vergleichende Morphologie der Genitalorgane und Phylogenie der liphistiomorphen Webspinnen (Araneae: Mesothelae). I. Revision der bisher bekannten Arten. 
Zeitschrift Fur Zoologische Systematik Und Evolutionsforschung 21: 275-293. https:// doi.org/10.1111/j.1439-0469.1983.tb00296.x

Haupt J (1984) Comportement sexuel, morphologie génitale et phylogenèse des araignées liphistiomorphes. Revue Arachnologique 5(4): 161-168.

Haupt J (2003) The Mesothelaea - monograph of an exceptional group of spiders (Araneae: Mesothelae) (Morphology, behaviour, ecology, taxonomy, distribution and phylogeny). Zoologica 154: 1-102.

Kishida K (1920) Zoological Magazine. Tokyo 32: 1-362. [no English title]

Kishida K (1923) Heptathela, a new genus of liphistiid spiders. Annotationes Zoologicae Japonenses 10: 235-242.

Ono H (1996) Two new species of the families Liphistiidae and Thomisidae (Araneae) from the Ryukyu Islands, southwest Japan. Acta Arachnologica Tokyo 45: 157-162. https://doi. org/10.2476/asjaa.45.157

Ono H (1997) A new species of the genus Heptathela (Araneae: Liphistiidae) from Vietnam. Acta Arachnologica 46: 23-28. https://doi.org/10.2476/asjaa.46.23

Ono H (1998) Spiders of the genus Heptathela (Araneae, Liphistiidae) from Kyushu, Japan. Memoirs of the National Science Museum Tokyo 30: 13-27.

Ono H (1999) Spiders of the genus Heptathela (Araneae, Liphistiidae) from Vietnam, with notes on their natural history. Journal of Arachnology 27: 37-43. https://doi. org/10.1080/096708799228085

Ono H (2010) Four new spiders (Arachnida, Araneae) of the families Liphistiidae, Ctenizidae, Araneidae and Ctenidae from Vietnam. Memoirs of the National Museum of Nature and Science Tokyo 46: 1-12.

Ono H (2002) New and remarkable spiders of the families Liphistiidae, Argyronetidae, Pisauridae, Theridiidae and Araneidae (Arachnida) from Japan. Bulletin of the National Museum of Nature and Science Tokyo (A) 28: 51-60.

Ono H (2009) The spiders of Japan with keys to the families and genera and illustrations of the species. Tokai University Press, Kanagawa, 739 pp.

Ono H, Nishikawa Y (1989) Taxonomic revision of the heptathelid spider (Araneae, Mesothelae) from Amami-ôshima Island, the Ryukyus. Memoirs of the National Science Museum Tokyo 22: 119-125.

Ono H, Ogata K (2018) Spiders of Japan, their natural history and diversity. Tokai University Press, Kanagawa, 714 pp.

Planas E, Ribera C (2015) Description of six new species of Loxosceles (Araneae: Sicariidae) endemic to the Canary Islands and the utility of DNA barcoding for their fast and accurate identification. Zoological Journal of the Linnean Society 174: 47-73. https://doi. org/10.1111/zoj.12226

Sawaguti Y, Ozi Y (1937) On Heptathela kimurai (Kishida, 1920). Acta Arachnologica 2: 115123. https://doi.org/10.2476/asjaa.2.115

Schenkel E (1953) Chinesische Arachnoidea aus dem Museum Hoangho-Peiho in Tientsin. Boletim do Museu Nacional do Rio de Janeiro (N.S., Zool.) 119: 1-108.

Schiödte JC (1849) Om en afigende sloegt af spindlernes orden. Naturhistorisk Tidsskrift 2: 617-624. 
Schwendinger PJ, Ono H (2011) On two Heptathela species from southern Vietnam, with a discussion of copulatory organs and systematics of the Liphistiidae (Araneae: Mesothelae). Revue suisse de Zoologie 118: 599-637. https://doi.org/10.5962/bhl.part.117818

Song DX, Haupt J (1984) Comparative morphology and phylogeny of liphistiomorph spiders (Araneae: Mesothelae) 2. Revision of new Chinese heptathelid species. Verhandlungen des Naturwissenschaftlichen Vereins in Hamburg 27: 443-451.

Song DX, Wu KY (1997) On a new species of the genus Heptathela (Araneae: Liphistiidae) from Hong Kong, China. Chinese Journal of Zoology 32: 1-3.

Tanikawa A (2013) Taxonomic revision of the spider genus Ryuthela (Araneae: Liphistiidae). Acta Arachnologica 62: 33-40. https://doi.org/10.2476/asjaa.62.33

Tanikawa A, Miyashita T (2014) Discovery of a cryptic species of Heptathela from the northernmost part of Okinawajima Is., Southwest Japan, as revealed by mitochondrial and nuclear DNA. Acta Arachnologica 63(2): 65-72. https://doi.org/10.2476/asjaa.63.65

Wang HZ, Jiao YC (1995) A new species of the family [sic] Heptathela in China. Journal of Yunan Normal University 15(1): 80-81.

Wang JF (1989) A new species of spider of the genus Liphistius from south China (Araneae: Liphistiidae). Acta Zootaxonomica Sinica 14: 30-32.

World Spider Catalog (2019) World Spider Catalog. Natural History Museum Bern. http:// wsc.nmbe.ch, version 20.0. [accessed on 12 July 2019]

Xu X, Liu FX, Cheng RC, Chen J, Xu X, Zhang ZS, Ono H, Pham DS, Norma-Rashid Y, Arnedo MA, Kuntner M, Li D (2015a) Extant primitively segmented spiders have recently diversified from an ancient lineage. Proceedings of the Royal Society of London B 282: 20142486. https://doi.org/10.1098/rspb.2014.2486

Xu X, Liu FX, Chen J, Ono H, Li D, Kuntner M (2015b) A genus-level taxonomic review of primitively segmented spiders (Mesothelae: Liphistiidae). ZooKeys 488: 121-151. https:// doi.org/10.3897/zookeys.488.8726

Xu X, Liu FX, Chen J, Li D, Kuntner M (2015c) Integrative taxonomy of the primitively segmented spider genus Ganthela (Araneae: Mesothelae: Liphistiidae) - DNA barcoding gap agrees with morphology. Zoological Journal of the Linnean Society 175: 288-306. https:// doi.org/10.1111/zoj.12280

Xu X, Liu FX, Chen J, Ono H, Li D, Kuntner M (2016) Pre-Pleistocene geological events shaping diversification and distribution of primitively segmented spiders on East Asian Margins. Journal of Biogeography 43: 1004-1019. https://doi.org/10.1111/jbi.12687

Xu X, Liu FX, Ono H, Chen J, Kuntner M, Li D (2017) Targeted sampling in Ryukyus facilitates species delimitation of the primitively segmented spider genus Ryuthela (Araneae: Mesothelae: Liphistiidae). Zoological Journal of the Linnean Society 181(4): 867-909. https://doi.org/10.1093/zoolinnean/zlx024

Xu X, Kuntner M, Ono H, Liu FX, Li D (2019) A multi-tier species delimitation approach resolves conflicts in delineating the primitively segmented spider genus Heptathela endemic to Japanese islands. bioRxiv. https://doi.org/10.1101/812214

Xu X, Yin CM (2001) A new species of the genus Heptathela from China (Araneae: Liphistiidae). Acta Arachnologica Sinica 10(1): 8-10.

Yaginuma T (1954) Synopsis of Japanese spiders (1). Atypus 5: 13-24. 
Yaginuma T (1955) The development of araneology in Japan. Annual Report of the Otemon Gakuin University 1: 25-41.

Yaginuma T (1960) Spiders of Japan in colour. Hoikusha, Osaka, 186 pp.

Yaginuma T (1971) Spiders of Japan in colour (enlarged and revised edition). Hoikusha, Osaka (for 1969), 197 pp.

Yaginuma T (1979) Heptathela nishihirai Haupt, 1979 from Okinawa. Atypus 75: 1-2.

Yaginuma T (1980) A supplementary note on "Heptathela nishihirai" (Araneae: Heptathelidae). Atypus 76: 44-45.

Yaginuma T (1986) Spiders of Japan in color (new edn.). Hoikusha Publishing Co., Osaka, 305 pp. [64 pls.]

Yin CM (2001) A new species of the genus Heptathela and its variant type from China (Araneae: Liphistiidae). Acta Zootaxonomica Sinica 26: 297-300.

Yin CM, Tang G, Xu X (2003) Two new species of the genus Heptathela from China (Araneae: Liphistiidae). Acta Arachnologica Sinica 12: 1-5.

Yin CM, Tang G, Zhao JZ, Chen J (2002) Two new species of the genus Heptathela from China (Araneae: Liphistiidae). Acta Arachnologica Sinica 11: 18-21.

Yoo JC, Kim JP (2002) Studies on basic pattern and evolution of male palpal organ (Arachnida: Araneae). Korean Arachnology 18: 13-31.

Yoshikura M (1955) Embryological studies on the liphistiids spider Heptathela kimurai. Part II. Kumamoto Journal of Science B 2: 1-86.

Yoshikura M (1983) Sexual characters in a liphistiid spider, Heptathela kimurai (Araneae: Heptathelidae). Heptathela 2: 63-73.

Yoshikura M (1987) The biology of spiders. Japan Scientific Societies Press, Tokyo, 613 pp.

Zhu CD, Wang YW (1984) A new species of genus Liphistius (Araneae: Liphistiidae). Journal of the Bethune Medical University 10: 251-253.

\section{Supplementary material I}

\section{Table S1}

Authors: Xin Xu, Hirotsugu Ono, Matjaž Kuntner, Fengxiang Liu, Daiqin Li

Data type: species data

Copyright notice: This dataset is made available under the Open Database License (http://opendatacommons.org/licenses/odbl/1.0/). The Open Database License $(\mathrm{ODbL})$ is a license agreement intended to allow users to freely share, modify, and use this Dataset while maintaining this same freedom for others, provided that the original source and author(s) are credited.

Link: https://doi.org/10.3897/zookeys.888.34494.suppl1 